

\title{
Poincaré-Birkhoff-Witt deformations of smash product algebras from Hopf actions on Koszul algebras
}

\author{
Chelsea Walton and Sarah Witherspoon
}

Let $H$ be a Hopf algebra and let $B$ be a Koszul $H$-module algebra. We provide necessary and sufficient conditions for a filtered algebra to be a Poincaré-BirkhoffWitt (PBW) deformation of the smash product algebra $B \# H$. Many examples of these deformations are given.

A correction was posted on 3 May 2017 in an online supplement.

\section{Introduction}

Given a Hopf algebra $(H$-)action on a Koszul algebra $B$, the aim of this work is to provide necessary and sufficient conditions for a certain filtered algebra, namely $\mathscr{D}_{B, \kappa}$ in Notation 0.3 below, to be a Poincaré-Birkhoff-Witt $(P B W)$ deformation of the smash product algebra $B \# H$, i.e., gr $\mathscr{D}_{B, \kappa} \cong B \# H$ (Definition 0.1). One wellstudied case is that of group actions on polynomial rings, where many algebras of interest arise as such deformations; see for example [Crawley-Boevey and Holland 1998; Drinfeld 1986; Etingof and Ginzburg 2002; Lusztig 1989; Ram and Shepler 2003; Shepler and Witherspoon 2012a]. For group actions on other Koszul algebras, see [Levandovskyy and Shepler 2014; Naidu and Witherspoon 2014; Shepler and Witherspoon 2012b; Shroff 2014]. There are some results involving Hopf algebra actions, such as those of Khare [2007], when $H$ is cocommutative and $B$ is a polynomial algebra. More specifically, the case when $H=U(\mathfrak{g})$, with $\mathfrak{g}$ the Lie algebra of a (not necessarily connected) reductive algebraic group, was studied by Etingof, Gan, and Ginzburg [Etingof et al. 2005], and by Khare and Tikaradze [2010] where $\mathfrak{g}=\mathfrak{s l}_{2}$. Results for an action of the quantized enveloping algebra $H=U_{q}\left(\mathfrak{s l}_{2}\right)$ on the quantum plane are provided by Gan and Khare [2007].

The goal of this paper is to provide a general theorem encompassing all of the above known classes of examples from the literature. Specifically, Theorem 3.1 gives PBW deformation conditions for $B \# H$, and it only requires the following

MSC2010: primary 16S80; secondary 16E40, 16S37, 16S40.

Keywords: Hopf algebra, Koszul algebra, PBW deformation, smash product algebra. 
of $H$ and $B$ : (1) the antipode of the Hopf algebra $H$ is bijective, (2) the Koszul $H$-module algebra $B$ is connected $\left(B_{0}=k\right)$, and (3) the $H$-action on $B$ preserves the grading of $B$. We then apply our theorem to several different choices of Hopf algebras acting on Koszul algebras to obtain nontrivial PBW deformations, both known and new. Our work indicates that such examples abound.

Many ring-theoretic properties are preserved under PBW deformation. To discuss this, let us consider the following definition:

Definition 0.1. Let $D=\bigcup_{i \geq 0} F_{i}$ be a filtered algebra with $\{0\} \subseteq F_{0} \subseteq F_{1} \subseteq \cdots \subseteq D$. We say that $D$ is a Poincaré-Birkhoff-Witt $(P B W)$ deformation of an $\mathbb{N}$-graded algebra $A$ if $A$ is isomorphic to the associated graded algebra $\operatorname{gr}_{F} D=\bigoplus_{i \geq 0} F_{i} / F_{i-1}$, as $\mathbb{N}$-graded algebras.

Now if $\operatorname{gr}_{F} D$ is an integral domain, prime, or (right) noetherian, then so is $D$. Moreover, if $D$ is affine with the standard filtration $F^{\prime}$, then the Gelfand-Kirillov (GK) dimensions of $D$ and of $\operatorname{gr}_{F^{\prime}} D$ are equal; $\operatorname{GKdim}\left(\operatorname{gr}_{F} D\right) \leq \operatorname{GKdim}(D)$ for a general filtration $F$ of $D$. The Krull dimension and global dimension of $\operatorname{gr}_{F} D$ serve as upper bounds for the corresponding dimensions of $D$. These ring-theoretic results can all be found in [McConnell and Robson 2001]. Homological properties preserved under PBW deformation have also been investigated; see [Berger and Taillefer 2007] and [Wu and Zhu 2013] regarding the Calabi-Yau property, for instance. The representation theory of some classes of PBW deformations of smash product algebras has been thoroughly studied in the literature and still remains an active area of research. Some examples of PBW deformations whose representation theory is of interest include rational Cherednik algebras, symplectic reflection algebras, and various types of Hecke algebras (see, for example, [Drinfeld 1986; Etingof et al. 2005; Etingof and Ginzburg 2002; Lusztig 1989; Ram and Shepler 2003], and for more recent work, see [Ding and Tsymbaliuk 2013; Losev and Tsymbaliuk 2014; Tikaradze 2010; Tsymbaliuk 2014]).

In order to state the main result, we need the following notation and terminology. Let $k$ be a field of arbitrary characteristic and let an unadorned $\otimes$ mean $\otimes_{k}$. Let $\mathbb{N}$ denote the natural numbers, including 0 . Recall that an $\mathbb{N}$-graded algebra is Koszul if its trivial module $k$ admits a linear minimal graded free resolution; see [Polishchuk and Positselski 2005, Chapter 2] for more details.

Notation $0.2\left(H, B, I, \kappa, \kappa^{C}, \kappa^{L}\right)$. Let $V$ be a finite-dimensional vector space over $k$.

(i) Let $H$ be a Hopf algebra with the standard structure notation $(H, m, \Delta, u, \epsilon, S)$. Here, we assume that the antipode $S$ of $H$ is bijective.

(ii) Let $B=T_{k}(V) /(I)$ be an $\mathbb{N}$-graded, Koszul, left $H$-module algebra $B=$ $\bigoplus_{j \geq 0} B_{j}$ with $B_{0}=k$ and $I \subseteq V \otimes V$. We assume that the action of $H$ preserves the grading and the subspace $I$ of $V \otimes V$. So in this case, $V$ is an $H$-module. 
(iii) Take $\kappa: I \rightarrow H \oplus(V \otimes H)$ to be a $k$-bilinear map, where $\kappa$ is the sum of its constant and linear parts $\kappa^{C}: I \rightarrow H$ and $\kappa^{L}: I \rightarrow V \otimes H$, respectively.

Notation $0.3\left(\mathscr{D}_{B, \kappa}\right)$. Let $\mathscr{D}_{B, \kappa}$ be the filtered $k$-algebra given by

$$
\mathscr{D}_{B, \kappa}=\frac{T_{k}(V) \# H}{(r-\kappa(r))_{r \in I}} .
$$

Here, we assign the elements of $H$ degree 0 .

Our main result is given as follows:

Theorem 3.1. The algebra $\mathscr{D}_{B, \kappa}$ is a PBW deformation of $B \# H$ if and only if the following conditions hold:

(a) $\kappa$ is $H$-invariant (Definition 1.4$)$; and

If $\operatorname{dim}_{k} V \geq 3$, then the following equations hold for the maps $\kappa^{C} \otimes \mathrm{id}-\mathrm{id} \otimes \kappa^{C}$ and $\kappa^{L} \otimes \mathrm{id}-\mathrm{id} \otimes \kappa^{L}$, which are defined on the intersection $(I \otimes V) \cap(V \otimes I)$ :

(b) $\operatorname{Im}\left(\kappa^{L} \otimes \mathrm{id}-\mathrm{id} \otimes \kappa^{L}\right) \subseteq I$;

(c) $\kappa^{L} \circ\left(\kappa^{L} \otimes \mathrm{id}-\mathrm{id} \otimes \kappa^{L}\right)=-\left(\kappa^{C} \otimes \mathrm{id}-\mathrm{id} \otimes \kappa^{C}\right)$;

(d) $\kappa^{C} \circ\left(\mathrm{id} \otimes \kappa^{L}-\kappa^{L} \otimes \mathrm{id}\right) \equiv 0$.

In the case that $H$ is cocommutative and $B$ is the symmetric algebra $S(V)$, this result was proven by Khare [2007, Theorem 2.1], via the diamond lemma. Our proof is a generalization of that of [Braverman and Gaitsgory 1996, Lemma 0.4, Theorem 0.5] (where $H=k$ ) and of [Shepler and Witherspoon 2012b, Theorem 5.4] (where $H$ is a group algebra).

Background information on Hopf algebra (co)actions, Hochschild cohomology, and deformations of algebras are provided in Section 1. In Section 2, we produce a free resolution of the smash product algebra $B \# H$; see Construction 2.5 and Theorem 2.10. This resolution is adapted from Guccione and Guccione [2002]; Negron [2014] independently constructed a similar resolution. Our resolution is used in the proof of Theorem 3.1, which is given in Section 3. Many examples of PBW deformations of $B \# H$ are provided in Section 4, including/involving:

- (Example 4.1) the Crawley-Boevey-Holland algebras;

- (Examples 4.2 and 4.4) some actions of semisimple, noncommutative, noncocommutative Hopf algebras on skew polynomial rings;

- (Examples 4.13 and 4.16) actions of the Sweedler and the Taft algebras on the polynomial ring $k[u, v]$;

- (Example 4.18) the quantized symplectic oscillator algebras of rank 1.

All of the examples of $B \# H$ above have nontrivial PBW deformations. 


\section{Background material}

We begin by discussing Hopf (co)actions on algebras and (co)modules and end with a discussion on deformations of algebras. For further background on these topics, we refer the reader to [Montgomery 1993] and [Braverman and Gaitsgory 1996; Gerstenhaber 1964], respectively.

\section{A. Hopf algebra (co)actions.}

Definition 1.1. (i) For a left $H$-module $M$, we denote the $H$-action by $\cdot: H \otimes M \rightarrow$ $M$, that is, by $h \cdot m \in M$ for all $h \in H, m \in M$. Similarly for all $h \in H$ and $m \in M$, we denote the right $h$-action on $m$ by $m \cdot h$.

(ii) Given a Hopf algebra $H$ and an algebra $A$, we say that $H$ acts on $A$ (from the left, as a Hopf algebra) if $A$ is a left $H$-module and

$$
h \cdot(a b)=\sum\left(h_{1} \cdot a\right)\left(h_{2} \cdot b\right) \quad \text { and } \quad h \cdot 1_{A}=\epsilon(h) 1_{A}
$$

for all $h \in H, a, b \in A$, where the comultiplication is given by $\Delta(h)=\sum h_{1} \otimes h_{2}$ (Sweedler's notation). In this case, we say that $A$ is a left $H$-module algebra.

(iii) For any left $H$-comodule $M$, we denote the left $H$-coaction by $\rho(M) \subseteq H \otimes M$, where $\rho(m)=\sum m_{-1} \otimes m_{0}$ for $m_{-1} \in H$ and $m, m_{0} \in M$. Likewise, the right $H$ coaction on a right $H$-comodule $M$ is given by $\rho(m)=\sum m_{0} \otimes m_{1}$ for $m, m_{0} \in M$ and $m_{1} \in H$.

Note that $H$ is naturally an $H$-bimodule via left and right multiplication. This yields a left $H$-adjoint action on $H$ given by

$$
h \cdot \ell:=\sum h_{1} \ell S\left(h_{2}\right)
$$

for $h, \ell \in H$. Moreover, if $V$ is a left $H$-module, we give $V \otimes H$ an $H$-bimodule structure as follows: $h(v \otimes \ell)=\sum\left(h_{1} \cdot v\right) \otimes h_{2} \ell$ and $(v \otimes \ell) h=v \otimes \ell h$ for all $h, \ell \in H$ and $v \in V$. A left $H$-adjoint action on $V \otimes H$ arises by combining these:

$$
h \cdot(v \otimes \ell):=\sum\left(h_{1} \cdot v\right) \otimes h_{2} \ell S\left(h_{3}\right) .
$$

The left $H$-adjoint actions in (1-2) and (1-3) extend to the standard left $H$-adjoint action on $A=B \# H$ (where $B=T_{k}(V) /(I)$ as in Notation 0.2(ii)), via Definition 1.1, since the action of $H$ preserves $I$.

Now we discuss the $H$-invariance of the map $\kappa$ (Notation 0.2(iii)), which is one of the necessary conditions for the filtered algebra $\mathscr{D}_{B, \kappa}$ (Notation 0.3 ) to be a PBW deformation of $B \# H$.

Definition 1.4. Recall Notation 0.2. We say that the map $\kappa$ is $H$-invariant if $h \cdot(\kappa(r))=\kappa(h \cdot r)$ in $H \oplus(V \otimes H)$ for any relation $r \in I$ and $h \in H$. 
1B. Deformations of algebras and Hochschild cohomology. In this part, we remind the reader of the notion of a deformation of a $k$-algebra $A$ and how Hochschild cohomology plays a role in its construction. This is seminal work of Gerstenhaber [1964], adapted to our graded setting as in [Braverman and Gaitsgory 1996].

Definition $1.5\left(A_{t}, A_{(j)}\right)$. Let $A$ be an associative algebra and let $t$ be an indeterminate. A deformation of $A$ over $k[t]$ is an associative $k[t]$-algebra $A_{t}$ over $k[t]$ which is isomorphic to $A[t]$ as $k$-vector spaces, with multiplication given by

$$
a_{1} * a_{2}=\mu_{0}\left(a_{1} \otimes a_{2}\right)+\mu_{1}\left(a_{1} \otimes a_{2}\right) t+\mu_{2}\left(a_{1} \otimes a_{2}\right) t^{2}+\cdots
$$

for all $a_{1}, a_{2} \in A$. Here, $\mu_{i}: A \otimes A \rightarrow A$ is a $k$-linear map, referred to as the $i$-th multiplication map. Moreover, $\mu_{0}\left(a_{1} \otimes a_{2}\right)=a_{1} a_{2}$ is the usual product in $A$.

Now assume that $A$ is graded by $\mathbb{N}$. A graded deformation of $A$ over $k[t]$ is an algebra $A_{t}$ as above, which is itself graded by $\mathbb{N}$, setting $\operatorname{deg}(t)=1$. The map $\mu_{i}$ is homogeneous of degree $-i$ in this case. A $j$-th-level graded deformation of $A$ is a graded associative algebra $A_{(j)}$ over $k[t] /\left(t^{j+1}\right)$ that is isomorphic to $A[t] /\left(t^{j+1}\right)$ as $k$-vector spaces, with multiplication given by

$$
a_{1} * a_{2}=\mu_{0}\left(a_{1} \otimes a_{2}\right)+\mu_{1}\left(a_{1} \otimes a_{2}\right) t+\cdots+\mu_{j}\left(a_{1} \otimes a_{2}\right) t^{j} .
$$

The maps $\mu_{i}: A \otimes A \rightarrow A$ are extended to be linear over $k[t] /\left(t^{j+1}\right)$.

The associativity of $*$ for the deformation $A_{t}$ imposes conditions on the maps $\mu_{i}$. Specifically, for each degree $i$, the following equation must hold for all $a_{1}, a_{2}, a_{3} \in A$ :

$$
\sum_{j=0}^{i} \mu_{j}\left(\mu_{i-j}\left(a_{1} \otimes a_{2}\right) \otimes a_{3}\right)=\sum_{j=0}^{i} \mu_{j}\left(a_{1} \otimes \mu_{i-j}\left(a_{2} \otimes a_{3}\right)\right) .
$$

We use Hochschild cohomology to study these equations.

Definition $1.7(B .(A))$. Let $A$ be a $k$-algebra and let $M$ be an $A$-bimodule, or equivalently, an $A^{e}$-module. Here, $A^{e}:=A \otimes A^{\mathrm{op}}$. The Hochschild cohomology of $M$ is $\operatorname{HH}^{n}(A, M):=\operatorname{Ext}_{A^{e}}^{n}(A, M)$. Moreover, this cohomology may be derived from the bar resolution $B$. $(A)$ of the $A^{e}$-module $A$ :

$$
\text { B. }(A): \quad \cdots \stackrel{\delta_{3}}{\longrightarrow} A^{\otimes 4} \stackrel{\delta_{2}}{\longrightarrow} A^{\otimes 3} \stackrel{\delta_{1}}{\longrightarrow} A \otimes A \stackrel{\delta_{0}}{\longrightarrow} A \longrightarrow 0,
$$

where

$$
\delta_{n}\left(a_{0} \otimes \cdots \otimes a_{n+1}\right):=\sum_{i=0}^{n}(-1)^{i} a_{0} \otimes \cdots \otimes a_{i} a_{i+1} \otimes \cdots \otimes a_{n+1}
$$

for all $n \geq 0$ and $a_{0}, \ldots, a_{n+1} \in A$. When $M=A$, write $\operatorname{HH}^{n}(A)$ for $\operatorname{HH}^{n}(A, A)$. Moreover, if $A$ is $\left(\mathbb{N}\right.$-)graded, then $\operatorname{HH}^{n}(A)$ inherits the grading of $A$ : If $A=\bigoplus_{i} A_{i}$, then $\mathrm{HH}^{n}(A)=\bigoplus_{i} \mathrm{HH}^{n, i}(A)$. 
Note that $\operatorname{Hom}_{k}\left(A^{\otimes n}, A\right) \cong \operatorname{Hom}_{A^{e}}\left(A^{\otimes(n+2)}, A\right)$, since the $A^{e}$-module $A^{\otimes(n+2)}$ is induced from the $k$-module $A^{\otimes n}$. We will identify these two Hom spaces often without further comment. Now we make some remarks about the multiplication maps $\mu_{i}$.

Remark 1.8. Using (1-6) for $i=1$, we see that

$$
\mu_{1}\left(a_{1} \otimes a_{2}\right) a_{3}+\mu_{1}\left(a_{1} a_{2} \otimes a_{3}\right)=a_{1} \mu_{1}\left(a_{2} \otimes a_{3}\right)+\mu_{1}\left(a_{1} \otimes a_{2} a_{3}\right)
$$

for all $a_{1}, a_{2}, a_{3} \in A$. In other words, $\mu_{1}$ is a Hochschild 2-cocycle on the bar resolution of $A$; that is, $\delta_{3}^{*}\left(\mu_{1}\right):=\mu_{1} \circ \delta_{3}$ vanishes. (Here we have identified the input $a_{1} \otimes a_{2} \otimes a_{3}$ with $1 \otimes a_{1} \otimes a_{2} \otimes a_{3} \otimes 1$ to apply $\delta_{3}$, under the identification of Hom spaces described above.)

Next, using (1-6) for $i=2$, we see that

$$
\begin{aligned}
\mu_{2}\left(a_{1} \otimes a_{2}\right) a_{3}+\mu_{1}\left(\mu_{1}\left(a_{1} \otimes a_{2}\right) \otimes a_{3}\right)+\mu_{2}\left(a_{1} a_{2} \otimes a_{3}\right) \\
\quad=a_{1} \mu_{2}\left(a_{2} \otimes a_{3}\right)+\mu_{1}\left(a_{1} \otimes \mu_{1}\left(a_{2} \otimes a_{3}\right)\right)+\mu_{2}\left(a_{1} \otimes a_{2} a_{3}\right) .
\end{aligned}
$$

Therefore

$$
\delta_{3}^{*}\left(\mu_{2}\right)\left(a_{1} \otimes a_{2} \otimes a_{3}\right)=\mu_{1}\left(\mu_{1}\left(a_{1} \otimes a_{2}\right) \otimes a_{3}\right)-\mu_{1}\left(a_{1} \otimes \mu_{1}\left(a_{2} \otimes a_{3}\right)\right)
$$

for all $a_{1}, a_{2}, a_{3} \in A$. In other words, $\mu_{2}$ is a cochain on the bar resolution of $A$ whose coboundary is given by the right-hand side of (1-10).

For all $i \geq 1,(1-6)$ is equivalent to

$(1-11) \delta_{3}^{*}\left(\mu_{i}\right)\left(a_{1} \otimes a_{2} \otimes a_{3}\right)=\sum_{j=1}^{i-1} \mu_{j}\left(\mu_{i-j}\left(a_{1} \otimes a_{2}\right) \otimes a_{3}\right)-\mu_{j}\left(a_{1} \otimes \mu_{i-j}\left(a_{2} \otimes a_{3}\right)\right)$.

That is, $\mu_{i}$ is a cochain on the bar resolution of $A$ whose coboundary is given by the right-hand side of (1-11).

Definition 1.12. The right-hand side of (1-11) is the $(i-1)$-th obstruction of the deformation $A_{t}$ of $A$ from Definition 1.5. An $(i-1)$-th-level graded deformation (defined by maps $\left.\mu_{1}, \ldots, \mu_{i-1}\right)$ lifts to an $i$-th-level graded deformation if there exists a map $\mu_{i}$ for which $\mu_{1}, \ldots, \mu_{i-1}, \mu_{i}$ define an $i$-th-level graded deformation.

The next proposition makes clear the choice of terminology in the above definition. Ultimately, one is interested in a deformation of $A$ over $k[t]$ and its specializations at particular values of $t$. The $i$-th-level graded deformations are steps in this direction.

Proposition 1.13 [Braverman and Gaitsgory 1996, Proposition 1.5]. All obstructions to lifting an $(i-1)$-th-level graded deformation to the next level lie in $\mathrm{HH}^{3,-i}(A)$. An $(i-1)$-th-level deformation lifts to the $i$-th-level if and only if its $(i-1)$-th obstruction cocycle is zero in cohomology, i.e., there is a map $\mu_{i}$ such that (1-11) holds for all $a_{1}, a_{2}, a_{3}$ in A. 
The connection between graded deformations and PBW deformations is well known; the following statement is a consequence of the canonical embedding of $A$ as a $k$-linear direct summand of $A[t]$, with splitting map given by specialization at $t=0$.

Proposition 1.14 [Braverman and Gaitsgory 1996, Remark 1.4]. Given a graded algebra $A$ and a graded deformation $A_{t}$ of $A$, then $A_{t}$ specialized at $t=1$ is a PBW deformation of $A$.

Now we explain that the two notions of deformation of $B \# H$ coincide; recall Notations 0.2 and 0.3. The following result is well known in related contexts, but we include some details for the reader's convenience.

Proposition 1.15. The following statements are equivalent.

- The algebra $\mathscr{D}_{B, \kappa}:=\left(T_{k}(V) \# H\right) /(r-\kappa(r))_{r \in I}$ is a PBW deformation of $B \# H$.

- The algebra $\mathscr{D}_{B, \kappa, t}:=\left(T_{k}(V) \# H\right)[t] /\left(r-\kappa^{L}(r) t-\kappa^{C}(r) t^{2}\right)_{r \in I}$ is a graded deformation of $B \# H$ over $k[t]$.

Proof. Assume that $\mathscr{D}_{B, \kappa}$ is a PBW deformation of $B \# H$. By its definition, $\mathscr{D}_{B, \kappa, t}$ is an associative algebra, and so we need only see that it is isomorphic to $B \# H[t]$ as a vector space. To this end, use the PBW property to define a $k$-linear map $\pi: B \# H \rightarrow T_{k}(V) \# H$ whose composition with the quotient map onto $\mathscr{D}_{B, \kappa}$ is an isomorphism of filtered vector spaces. Extend $\pi$ to a $k[t]$-linear map from $B \# H[t]$ to $T_{k}(V) \# H[t]$. Its composition with the quotient map to $\mathscr{D}_{B, \kappa, t}$ is an isomorphism of $k$-vector spaces; one sees this by a degree argument.

Conversely, assume that $\mathscr{D}_{B, \kappa, t}$ is a graded deformation of $B \# H$ over $k[t]$. We may specialize to $t=1$ to obtain $\mathscr{D}_{B, \kappa}$. Now apply Proposition 1.14 to conclude that $\mathscr{D}_{B, \kappa}$ is a PBW deformation of $B \# H$.

\section{Resolutions for smash product algebras}

In this section, let $A$ denote the smash product $B \# H$, which is an $\mathbb{N}$-graded algebra: $A=\bigoplus_{j \geq 0}\left(B_{j} \otimes H\right)$. Thus $A_{0} \cong H$. The aim is to construct a free $A^{e}$-resolution $X$. of the $A^{e}$-module $A$ from resolutions of $H$ and of $B$ (denoted by $C_{\bullet}$ and $D_{\bullet}$, respectively). This construction simultaneously generalizes results of Guccione and Guccione [2002] and of Shepler and Witherspoon [2012b, Section 4]. A similar resolution was constructed independently by Negron [2014].

Definition 2.1 $\left(C_{\bullet}, C_{i}, C_{i}^{\prime}\right)$. For $i \geq 0$, let $C_{i}$ denote the $H^{e}$-module $H^{\otimes(i+2)}$. The left $H$-comodule structure $\rho: C_{i} \rightarrow H \otimes C_{i}$ is given by

$$
\rho\left(h^{0} \otimes h^{1} \otimes \cdots \otimes h^{i+1}\right):=\sum h_{1}^{0} \cdots h_{1}^{i+1} \otimes h_{2}^{0} \otimes \cdots \otimes h_{2}^{i+1} \in H \otimes C_{i}
$$

for all $h^{0}, \ldots, h^{i+1} \in H$. For $h \in H$, the left and right $h$-actions on an element $x \in C_{i}$ are given respectively by left and right multiplication by $h$ in the leftmost and rightmost factors of $x$. Now, let 


$$
C_{.}: \quad \cdots \longrightarrow C_{1} \longrightarrow C_{0} \longrightarrow H \longrightarrow 0
$$

be the bar resolution $\boldsymbol{B} .(H)$ of $H$ (Definition 1.7), which is an $H^{e}$-free resolution of $H$.

There is an isomorphism of free $H^{e}$-modules $C_{i} \cong H \otimes C_{i}^{\prime} \otimes H$, where $C_{i}^{\prime}=H^{\otimes i}$ if $i \geq 1$ and $C_{0}^{\prime}=k$. We give each $C_{i}^{\prime}$ the $H$-comodule structure inducing that on $C_{i}$ under the usual tensor product of comodules.

Remark 2.2. The resolution $C$. satisfies the following conditions:

(i) The right $H$-action and left $H$-coaction on $C_{i}$ commute in the sense that for all $x \in C_{i}$ and $h \in H$

$$
\sum(x \cdot h)_{-1} \otimes(x \cdot h)_{0}=\sum x_{-1} h_{1} \otimes\left(x_{0} \cdot h_{2}\right) .
$$

That is, each $C_{i}$ is a Hopf module (for which the action is a left action and the coaction is a right coaction).

(ii) The differentials are left $H$-comodule homomorphisms.

Definition $2.3\left(D_{.}, D_{i}, D_{i}^{\prime}\right)$. Recall that $B$ is a Koszul algebra. Let

$$
\cdots \longrightarrow D_{1} \longrightarrow D_{0} \longrightarrow B \longrightarrow 0
$$

be the Koszul resolution of $B$ as a $B^{e}$-module: $D_{0}=B \otimes B, D_{1}=B \otimes V \otimes B$, $D_{2}=B \otimes I \otimes B$, and for each $n \geq 3, D_{i}=B \otimes D_{i}^{\prime} \otimes B$, where

$$
D_{i}^{\prime}=\bigcap_{j=0}^{i-2}\left(V^{\otimes j} \otimes I \otimes V^{\otimes(i-2-j)}\right) .
$$

Each $D_{i}$ is a subspace of $B^{\otimes(i+2)}$, and the differential on the Koszul resolution is the one induced by the canonical embedding of the Koszul resolution into the bar resolution of $B$.

Remark 2.4. The resolution $D$. satisfies the following conditions:

(i) Each $B^{e}$-module $D_{i}$ is a left $H$-module and the differentials are $H$-module homomorphisms.

(ii) The left actions of $B$ and $H$ on $D_{i}$ are compatible in the sense that they induce a left action of $A=B \# H$ on $D_{i}$.

(iii) In addition, the right $B$-action on $D_{i}$ is compatible with the left $H$-action on $D_{i}$ in the sense that for all $h \in H, y=b^{0} \otimes y^{\prime} \otimes b^{1} \in D_{i}$ for $y^{\prime} \in D_{i}^{\prime}$ and $b^{0}, b^{1}, b \in B$,

$$
\begin{aligned}
h \cdot(y \cdot b) & =\sum\left(h_{1} \cdot y\right) \cdot\left(h_{2} \cdot b\right)=\left[\sum\left(h_{1} \cdot b^{0}\right) \otimes\left(h_{2} \cdot y^{\prime}\right) \otimes\left(h_{3} \cdot b^{1}\right)\right] \cdot\left(h_{4} \cdot b\right) \\
& =\sum\left(h_{1} \cdot b^{0}\right) \otimes\left(h_{2} \cdot y^{\prime}\right) \otimes\left(h_{3} \cdot b^{1}\right) b=(h \cdot y) \cdot b .
\end{aligned}
$$


(iv) Each $D_{i}$ is considered to be a left $H$-comodule in a trivial way by requiring that it be $H$-coinvariant; that is, the comodule structure is given by maps $\rho_{i}: D_{i} \rightarrow H \otimes D_{i}$, where $\rho_{i}(y)=1 \otimes y$ for all $y \in D_{i}$. The maps $\rho_{i}$ are maps of left $H$-modules, if we give $H \otimes D_{i}$ the tensor product $H$-module structure, where the factor $H$ has the adjoint $H$-module structure. See Section 1A.

Construction $2.5\left(X_{\bullet}\right)$. We wish to combine the two resolutions, $C$. and $D$. from Definitions 2.1 and 2.3, to form a resolution $X$. of $A=B \# H$ by $A$-bimodules, via a tensor product. To that end, we first apply $\left(A \otimes_{H}-\right)$ to $C_{\text {. }}$ Note that $A$ is free as a right $H$-module (under multiplication) and that $A \otimes_{H} H \cong A$. The following sequence of $A \otimes H^{\mathrm{op}}$-modules is therefore exact:

$$
\cdots \longrightarrow A \otimes_{H} C_{1} \longrightarrow A \otimes_{H} C_{0} \longrightarrow A \longrightarrow 0 .
$$

Similarly, we apply $\left(-\otimes_{B} A\right)$ to $D$. Note that $A$ is free as a left $B$-module, and that $B \otimes_{B} A \cong A$. The following sequence of $B \otimes A^{\text {op }}$-modules is therefore exact:

$$
\cdots \longrightarrow D_{1} \otimes_{B} A \longrightarrow D_{0} \otimes_{B} A \longrightarrow A \longrightarrow 0 .
$$

We will next extend the actions on the modules in each of these two sequences so that they become $A^{e}$-modules. Then, we will take their tensor product over $A$.

We extend the right $H$-module structure on $A \otimes_{H} C$. to a right $A$-module structure by defining a right action of $B$ on $A \otimes_{H} C$.: for all $a \in A, x \in C_{i}, b \in B$, we set

$$
\left(a \otimes_{H} x\right) \cdot b:=\sum a\left(x_{-1} \cdot b\right) \otimes_{H} x_{0} .
$$

This does indeed make $A \otimes_{H} C_{i}$ into a right $B$-module, and, by combining with the right action of $H$, gives a right action of $A$ on $A \otimes_{H} C_{i}$. Note that for $x=x^{0} \otimes \cdots \otimes x^{i+1} \in C_{i}\left(\right.$ with $\left.x^{0}, \ldots, x^{i+1} \in H\right)$,

$$
\begin{aligned}
\rho(h x) & =\sum(h x)_{-1} \otimes(h x)_{0}=\sum h_{1} x_{1}^{0} \cdots x_{1}^{i+1} \otimes h_{2} x_{2}^{0} \otimes \cdots \otimes x_{2}^{i+1} \\
& =\sum h_{1} x_{-1} \otimes h_{2} x_{0} .
\end{aligned}
$$

The action is well-defined: If $h \in H$, then

$\left(a h \otimes_{H} x\right) \cdot b \stackrel{(2-6)}{=} \sum a h\left(x_{-1} \cdot b\right) \otimes_{H} x_{0}=\sum a\left(h_{1} x_{-1} \cdot b\right) \otimes_{H} h_{2} x_{0} \stackrel{(2-6)}{=}\left(a \otimes_{H} h x\right) \cdot b$.

Since the differentials on $C$. are $H$-comodule homomorphisms (Remark 2.2(ii)), this action commutes with the differentials.

We extend the left $B$-module structure on $D_{i} \otimes_{B} A$ to a left $A$-module structure by defining a left action of $H$ by

$$
h \cdot\left(y \otimes_{B} a\right):=\sum\left(h_{1} \cdot y\right) \otimes_{B} h_{2} a
$$


for all $h \in H, y \in D_{i}, a \in A$. It is well-defined, since for all $h \in H, b \in B$, we have by the definitions in Section $1 \mathrm{~A}$ that

$$
\begin{aligned}
h \cdot\left(y b \otimes_{B} a\right) & \stackrel{(2-7)}{=} \sum\left(h_{1} \cdot(y b)\right) \otimes_{B}\left(h_{2} a S\left(h_{3}\right)\right)=\sum\left(h_{1} \cdot y\right)\left(h_{2} \cdot b\right) \otimes_{B} h_{3} a S\left(h_{4}\right) \\
& =\sum\left(h_{1} \cdot y\right) \otimes_{B}\left(h_{2} \cdot b\right) h_{3} a S\left(h_{4}\right)=\sum\left(h_{1} \cdot y\right) \otimes_{B}\left(h_{2} \cdot(b a)\right) \\
& \stackrel{(2-7)}{=} h \cdot\left(y \otimes_{B} b a\right) .
\end{aligned}
$$

The left $H$-action on $D_{i}$ is compatible with the right $B$-action on $D_{i}$ by Remark 2.4(iii). Again, this action commutes with the differentials, since the differentials on $D$. are $H$-module homomorphisms (Remark 2.4(i)).

We may now consider $A \otimes_{H} C_{\mathbf{0}}$ and $D_{\bullet} \otimes_{B} A$ to be complexes of $A^{e}$-modules via the $A$-bimodule structure defined above. We take their tensor product over $A$, letting $X_{\bullet, \bullet}:=\left(A \otimes_{H} C_{\bullet}\right) \otimes_{A}\left(D_{\bullet} \otimes_{B} A\right)$; that is, for all $i, j \geq 0$,

$$
X_{i, j}:=\left(A \otimes_{H} C_{i}\right) \otimes_{A}\left(D_{j} \otimes_{B} A\right),
$$

with horizontal and vertical differentials

$$
d_{i, j}^{h}: X_{i, j} \rightarrow X_{i-1, j} \quad \text { and } \quad d_{i, j}^{v}: X_{i, j} \rightarrow X_{i, j-1}
$$

given by $d_{i, j}^{h}:=d_{i}^{C} \bullet \otimes \mathrm{id}$ and $d_{i, j}^{v}:=(-1)^{i} \mathrm{id} \otimes d_{j}^{D} \bullet$

Finally, let $X$. be the total complex of $X .$. :

$$
\cdots \longrightarrow X_{2} \longrightarrow X_{1} \longrightarrow X_{0} \longrightarrow A \longrightarrow 0,
$$

with $X_{n}=\bigoplus_{i+j=n} X_{i, j}$.

Theorem 2.10. We have the following statements:

(a) For each $i, j$, the $A^{e}$-module $X_{i, j}$ is isomorphic to $A \otimes C_{i}^{\prime} \otimes D_{j}^{\prime} \otimes A$.

(b) The complex $X$. given in (2-9) is a free resolution of the $A^{e}$-module A.

Proof. (a) Write $C_{i} \cong H \otimes C_{i}^{\prime} \otimes H$ and $D_{j} \cong B \otimes D_{j}^{\prime} \otimes B$ for vector spaces $C_{i}^{\prime}$ and $D_{j}^{\prime}$, as in Definitions 2.1 and 2.3. Then

$$
\begin{aligned}
X_{i, j} & \cong\left(A \otimes_{H} H \otimes C_{i}^{\prime} \otimes H\right) \otimes_{A}\left(B \otimes D_{j}^{\prime} \otimes B \otimes_{B} A\right) \\
& \cong\left(A \otimes C_{i}^{\prime} \otimes H\right) \otimes_{A}\left(B \otimes D_{j}^{\prime} \otimes A\right) .
\end{aligned}
$$

We will show that this is isomorphic to $A \otimes C_{i}^{\prime} \otimes D_{j}^{\prime} \otimes A$ as an $A^{e}$-module. First, define a map as follows:

$$
\left(A \otimes C_{i}^{\prime} \otimes H\right) \times\left(B \otimes D_{j}^{\prime} \otimes A\right) \rightarrow A \otimes C_{i}^{\prime} \otimes D_{j}^{\prime} \otimes A,
$$

$$
\left(a \otimes x \otimes h, b \otimes y \otimes a^{\prime}\right) \mapsto \sum a\left(x_{-1} h_{1} \cdot b\right) \otimes x_{0} \otimes\left(h_{2} \cdot y\right) \otimes h_{3} a^{\prime}
$$


for all $a, a^{\prime} \in A, x \in C_{i}^{\prime}, y \in D_{j}^{\prime}, h \in H, b \in B$. This map is $k$-bilinear by definition, and we will check that it is $A$-balanced. First, let $b^{\prime} \in B$. We rewrite $(a \otimes x \otimes h) \cdot b^{\prime}$ as follows. First, using $A \otimes C_{i}^{\prime} \otimes H \cong A \otimes_{H} C_{i}$, identify this element with $a \otimes_{H}(1 \otimes x \otimes h) \in A \otimes_{H} C_{i}$. By (2-6),

$$
\left(a \otimes_{H}(1 \otimes x \otimes h)\right) \cdot b^{\prime}=\sum a\left((1 \otimes x \otimes h)_{-1} \cdot b^{\prime}\right) \otimes_{H}(1 \otimes x \otimes h)_{0} .
$$

By Definition 2.1 , and by identifying $x \in C_{i}^{\prime}$ with $x^{1} \otimes x^{2} \otimes \cdots \otimes x^{i}$, we have that

$$
\begin{aligned}
\rho(1 \otimes x \otimes h) & =\sum(1 \otimes x \otimes h)_{-1} \otimes(1 \otimes x \otimes h)_{0} \\
& =\sum\left(x_{1}^{1} x_{1}^{2} \cdots x_{1}^{i} h_{1}\right) \otimes\left(1 \otimes x_{2}^{1} \otimes x_{2}^{2} \otimes \cdots \otimes x_{2}^{i} \otimes h_{2}\right) .
\end{aligned}
$$

So, $(1 \otimes x \otimes h)_{-1}=x_{-1} h_{1}$ and $(1 \otimes x \otimes h)_{0}=x_{0} \otimes h_{2}$. Now $C_{i} \cong H \otimes C_{i}^{\prime} \otimes H$ as an $H$-comodule, so

$$
\begin{aligned}
\left((a \otimes x \otimes h) \cdot b^{\prime}, b \otimes y \otimes a^{\prime}\right) & =\sum\left(a\left(x_{-1} h_{1} \cdot b^{\prime}\right) \otimes x_{0} \otimes h_{2}, b \otimes y \otimes a^{\prime}\right) \\
& \mapsto \sum a\left(x_{-2} h_{1} \cdot b^{\prime}\right)\left(x_{-1} h_{2} \cdot b\right) \otimes x_{0} \otimes\left(h_{3} \cdot y\right) \otimes h_{4} a^{\prime} .
\end{aligned}
$$

On the other hand,

$\left(\left(a \otimes x \otimes h, b^{\prime} \cdot\left(b \otimes y \otimes a^{\prime}\right)\right)=\left(a \otimes x \otimes h, b^{\prime} b \otimes y \otimes a^{\prime}\right)\right.$

$$
\left.\mapsto \sum a\left(x_{-1} h_{1} \cdot\left(b^{\prime} b\right)\right) \otimes x_{0} \otimes\left(h_{2} \cdot y\right) \otimes h_{3} a^{\prime}\right),
$$

which is the same as the previous image since $B$ is an $H$-module algebra. Now let $\ell \in H$. Then

$\left((a \otimes x \otimes h) \cdot \ell, b \otimes y \otimes a^{\prime}\right)=\left(a \otimes x \otimes h \ell, b \otimes y \otimes a^{\prime}\right)$

$$
\mapsto \sum a\left(x_{-1} h_{1} \ell_{1} \cdot b\right) \otimes x_{0} \otimes\left(h_{2} \ell_{2} \cdot y\right) \otimes h_{3} \ell_{3} a^{\prime} .
$$

On the other hand,

$$
\begin{aligned}
\left(a \otimes x \otimes h, \ell \cdot\left(b \otimes y \otimes a^{\prime}\right)\right) & =\sum\left(a \otimes x \otimes h,\left(\ell_{1} \cdot b\right) \otimes\left(\ell_{2} \cdot y\right) \otimes \ell_{3} a^{\prime}\right) \\
& \mapsto \sum a\left(x_{-1} h_{1} \ell_{1} \cdot b\right) \otimes x_{0} \otimes\left(h_{2} \ell_{2} \cdot y\right) \otimes h_{3} \ell_{3} a^{\prime},
\end{aligned}
$$

which is the same as the previous image. Therefore, there is an induced map

$$
\left(A \otimes C_{i}^{\prime} \otimes H\right) \otimes_{A}\left(B \otimes D_{j}^{\prime} \otimes A\right) \rightarrow A \otimes C_{i}^{\prime} \otimes D_{j}^{\prime} \otimes A .
$$

Now, we verify that the map below is an inverse map of (2-11):

$$
a \otimes x \otimes y \otimes a^{\prime} \mapsto(a \otimes x \otimes 1) \otimes_{A}\left(1 \otimes y \otimes a^{\prime}\right) .
$$

It is clear that first applying (2-12) and then (2-11) yields the identity map on $A \otimes C_{i}^{\prime} \otimes D_{j}^{\prime} \otimes A$. On the other hand, the image of first applying (2-11) then (2-12) to $\left(a \otimes x \otimes h, b \otimes y \otimes a^{\prime}\right)$ is 


$$
\begin{aligned}
\sum\left(a\left(x_{-1} h_{1} \cdot b\right) \otimes x_{0} \otimes 1\right) \otimes_{A}\left(1 \otimes\left(h_{2} \cdot y\right) \otimes h_{3} a^{\prime}\right) & \\
& =\sum\left(a\left(x_{-1} h_{1} \cdot b\right) \otimes x_{0} \otimes 1\right) \otimes_{A}\left(\epsilon\left(h_{2}\right) \otimes\left(h_{3} \cdot y\right) \otimes h_{4} a^{\prime}\right) \\
& =\sum\left(a\left(x_{-1} h_{1} \cdot b\right) \otimes x_{0} \otimes 1\right) \otimes_{A}\left(h_{2} \cdot\left(1 \otimes y \otimes a^{\prime}\right)\right) \\
& =\sum\left(a\left(x_{-1} h_{1} \cdot b\right) \otimes x_{0} \otimes h_{2}\right) \otimes_{A}\left(1 \otimes y \otimes a^{\prime}\right) \\
& \stackrel{(2-6)}{=}((a \otimes x \otimes h) \cdot b) \otimes_{A}\left(1 \otimes y \otimes a^{\prime}\right) \\
& =(a \otimes x \otimes h) \otimes_{A}\left(b \otimes y \otimes a^{\prime}\right) .
\end{aligned}
$$

Therefore the two $A^{e}$-modules $X_{i j}$ and $A \otimes C_{i}^{\prime} \otimes D_{i}^{\prime} \otimes A$ are isomorphic, as claimed. (b) We wish to apply the Künneth theorem to show that the complex $X_{\text {. is a }}$ free resolution of the $A^{e}$-module $A$. To that end, we check that each term in the complex $D_{0} \otimes_{B} A$ is a free left $A$-module and that the image of each differential in the complex is also projective as a left $A$-module. First, write each $D_{i} \otimes_{B} A \cong$ $\left(B \otimes D_{i}^{\prime} \otimes B\right) \otimes_{B} A \cong B \otimes D_{i}^{\prime} \otimes A$. Define a $k$-linear map $f: A \otimes D_{i}^{\prime} \otimes B \rightarrow B \otimes D_{i}^{\prime} \otimes A$ by

$$
f(r h \otimes y \otimes b)=\sum r \otimes\left(h_{1} \cdot y\right) \otimes h_{2} b
$$

for $h \in H, y \in D_{i}^{\prime}$, and $r, b \in B$. Give $A \otimes D_{i}^{\prime} \otimes B$ the structure of a left $A$-module by requiring $A$ to act by left multiplication on the leftmost factor. Clearly this is a free left $A$-module. The map $f$ is an $A$-module homomorphism by the definition of the left $A$-action on $B \otimes D_{i}^{\prime} \otimes A$; see (2-7). We claim that the following map is an inverse map, so that $f$ is an isomorphism of $A$-modules: let $S^{-1}$ denote the (composition) inverse of the antipode $S$ of $H$. Let $g: B \otimes D_{i}^{\prime} \otimes A \rightarrow A \otimes D_{i}^{\prime} \otimes B$ be the $k$-linear map defined by

$$
g(r \otimes y \otimes h b)=\sum r h_{2} \otimes\left(S^{-1}\left(h_{1}\right) \cdot y\right) \otimes b .
$$

Since for each $h \in H$ we have $\sum h_{2} S^{-1}\left(h_{1}\right)=\epsilon(h)=\sum S^{-1}\left(h_{2}\right) h_{1}$ (see, e.g., [Radford 2012, Proposition 7.1.10]), the function $g$ is indeed the inverse of $f$. Thus, each term in the complex $D_{\bullet} \otimes_{B} A$ is a free left $A$-module.

That the image of each differential is projective as a left $A$-module may be proved inductively, starting on one end of the complex

$$
\cdots \longrightarrow D_{1} \otimes_{B} A \stackrel{d_{1} \otimes \mathrm{id}}{\longrightarrow} D_{0} \otimes_{B} A \stackrel{d_{0} \otimes \mathrm{id}}{\longrightarrow} A \longrightarrow 0,
$$

as follows. Since $A$ is a projective left $A$-module and $d_{0} \otimes \mathrm{id}$ is surjective, the map splits, implying that $\operatorname{Ker}\left(d_{0} \otimes \mathrm{id}\right)=\operatorname{Im}\left(d_{1} \otimes \mathrm{id}\right)$ is a direct summand of the free left $A$-module $D_{0} \otimes_{B} A$. Therefore it is projective. Again, since $\operatorname{Im}\left(d_{1} \otimes \mathrm{id}\right)$ is projective, the map $d_{1} \otimes$ id from $D_{1} \otimes_{B} A$ to its image splits so that $\operatorname{Ker}\left(d_{1} \otimes \mathrm{id}\right)=\operatorname{Im}\left(d_{2} \otimes \mathrm{id}\right)$ 
is a direct summand of the free left $A$-module $D_{1} \otimes_{B} A$. Continuing in this way, we see that $\operatorname{Im}\left(d_{i} \otimes \mathrm{id}\right)$ is a free left $A$-module for each $i$.

The Künneth theorem [Weibel 1994, Theorem 3.6.3] then gives for each $n$ an exact sequence

$$
\begin{aligned}
& 0 \longrightarrow \bigoplus_{i+j=n} \mathrm{H}_{i}\left(A \otimes_{H} C_{\bullet}\right) \otimes_{A} \mathrm{H}_{j}\left(D_{\bullet} \otimes_{B} A\right) \longrightarrow \mathrm{H}_{n}\left(\left(A \otimes_{H} C_{\bullet}\right) \otimes_{A}\left(D_{\bullet} \otimes_{B} A\right)\right) \\
& \longrightarrow \bigoplus_{i+j=n-1} \operatorname{Tor}_{1}^{A}\left(\mathrm{H}_{i}\left(A \otimes_{H} C_{\bullet}\right), \mathrm{H}_{j}\left(D_{\bullet} \otimes_{B} A\right)\right) \rightarrow 0 .
\end{aligned}
$$

Now $A \otimes_{H} C_{\text {. }}$ and $D_{\bullet} \otimes_{B} A$ are exact except in degree 0 , where their homologies are each $A$; that is, $H_{0}\left(A \otimes_{H} C_{\bullet}\right)=A$ and $H_{0}\left(D_{\bullet} \otimes_{B} A\right)=A$. Therefore the only potentially nonzero Tor term is when $i=0=j$, or $\operatorname{Tor}_{1}^{A}(A, A)$, yet this equals 0 since $A$ is flat over $A$. So for each $n$, we have

$$
\mathrm{H}_{n}\left(\left(A \otimes_{H} C_{\bullet}\right) \otimes_{A}\left(D . \otimes_{B} A\right)\right) \cong \bigoplus_{i+j=n} \mathrm{H}_{j}\left(A \otimes_{H} C_{\bullet}\right) \otimes_{A} \mathrm{H}_{i}\left(D . \otimes_{B} A\right) .
$$

Again the right side is only nonzero when $i=0=j$, so we have

$$
\mathrm{H}_{0}\left(\left(A \otimes_{H} C_{\bullet}\right) \otimes_{A}\left(D . \otimes_{B} A\right)\right) \cong \mathrm{H}_{0}\left(A \otimes_{H} C_{\bullet}\right) \otimes_{A} \mathrm{H}_{0}\left(D_{\bullet} \otimes_{B} A\right) \cong A \otimes_{A} A \cong A
$$

and $\mathrm{H}_{n}\left(\left(A \otimes_{H} C_{\bullet}\right) \otimes_{A}\left(D_{\bullet} \otimes_{B} A\right)\right)=0$ for all $n>0$. Thus we have proven that $X$. is an $A^{e}$-free resolution of $A$.

We next relate the resolution $X$. of $A$ (from Construction 2.5) to the bar resolution B. $(A)$ of $A$ :

Lemma 2.13. There exist degree-preserving chain maps between $X$. and the bar resolution $\boldsymbol{B}$. (A) of $A$,

$$
\phi_{\bullet}: X_{\bullet} \longrightarrow \boldsymbol{B}_{\bullet}(A) \text { and } \psi_{\bullet}: \boldsymbol{B}_{\bullet}(A) \longrightarrow X_{\bullet},
$$

such that $\psi_{n} \phi_{n}$ is the identity map on the $A^{e}$-submodule $X_{0, n}$ of $X_{n}$ for each $n \geq 0$. Proof. Recall by Notation 0.2 that $B$ is generated by the vector space $V$, with quadratic relations $I \subseteq V \otimes V$. First we prove by induction on $n$ that there are degree-preserving maps $\phi_{n}: X_{n} \rightarrow A^{\otimes(n+2)}$ and $\psi_{n}: A^{\otimes(n+2)} \rightarrow X_{n}$ commuting with the differentials. For clarity, we denote the differential on the bar resolution of $A$ by $\delta$. We have the diagram

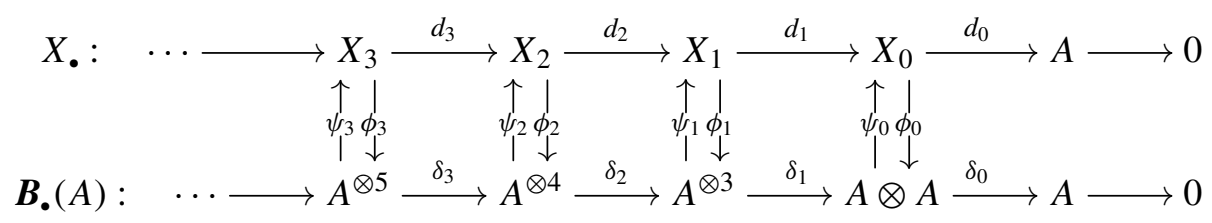

where $\boldsymbol{B}_{n}(A)=A^{\otimes(n+2)}$ and $X_{n}=\bigoplus_{i+j=n} X_{i, j}$, with $X_{i, j}$ defined in (2-8); see also Theorem 2.10(a). 
Define $\phi_{0}=\mathrm{id} \otimes \mathrm{id}=\psi_{0}$, the identity map from $A \otimes A$ to itself. We wish to define $\phi_{\bullet}$ so that when restricted to $X_{0, \bullet}$ it corresponds to the standard embedding of the Koszul complex into the bar complex: for $n=1$, this is the embedding of $A \otimes V \otimes A$ into $A \otimes A \otimes A$ via the containment of $V$ in $A$. We may define $\phi_{1}$ on $X_{1}=X_{0,1} \oplus X_{1,0} \cong(A \otimes V \otimes A) \oplus(A \otimes H \otimes A)$ by $\phi_{1}(1 \otimes v \otimes 1)=1 \otimes v \otimes 1$ and $\phi_{1}(1 \otimes h \otimes 1)=1 \otimes h \otimes 1$ for all $v \in V, h \in H$. Note that for $n \geq 2$,

$$
X_{0, n} \cong A \otimes\left(\bigcap_{i=0}^{n-2} V^{\otimes i} \otimes I \otimes V^{\otimes(n-i-2)}\right) \otimes A,
$$

which is a free $A^{e}$-submodule of $A^{\otimes(n+2)}$. For each $i, j$ with $i+j=n$, choose a basis of the vector space $C_{i}^{\prime} \otimes D_{j}^{\prime}$ (whose elements are homogeneous of degree $j$, as $H$ is declared to have degree 0 ). By hypothesis, $\phi_{n-1}$ is degree-preserving, and $d_{n}$ is degree-preserving by construction. So, applying $\phi_{n-1} d_{n}$ to these basis elements of $C_{i}^{\prime} \otimes D_{j}^{\prime}$ produces elements of degree $j$ in the kernel of $\delta_{n-1}$, that is, the image of $\delta_{n}$. We define $\phi_{n}$ by choosing (arbitrary) corresponding elements in the inverse image of $\operatorname{Im}\left(\delta_{n}\right)$. If we start with an element in $X_{0, n}$, we may choose its image in $A^{\otimes(n+2)}$ under the canonical embedding of $X_{0, n}$ into $A^{\otimes(n+2)}$ (see (2-14)). Given $X_{i, j}$ and $X_{i^{\prime}, j^{\prime}}$ with $i+j=i^{\prime}+j^{\prime}=n$ and $i \neq 0, i^{\prime} \neq 0$, elements of $X_{i, j}$ have degree $j$ and elements of $X_{i^{\prime}, j^{\prime}}$ have degree $j^{\prime}$. So their images under $\phi_{n}$ may be chosen independently, and in particular, independently of those of $X_{0, n}$. Thus, we have the maps $\phi_{n}$, as desired.

Now we show that $\psi_{n}$ may be chosen so that $\psi_{n} \phi_{n}$ is the identity map on $X_{0, n}$. In degree 1 , we have summands $X_{0,1} \cong A \otimes V \otimes A$ and $X_{1,0} \cong A \otimes H \otimes A$. Note that $V \oplus H$ is a direct summand of $A$ as a vector space. We may therefore define $\psi_{1}(1 \otimes v \otimes 1)=1 \otimes v \otimes 1$ in $X_{0,1}$ for all $v \in V$, and $\psi_{1}(1 \otimes h \otimes 1)=1 \otimes h \otimes 1$ in $X_{1,0}$ for all $h \in H$. We also have that $\psi_{1}$ is the identity map on elements of the form $1 \otimes z \otimes 1$, for $z$ ranging over a basis of a chosen complement of $V \oplus H$ as a vector subspace of $A$. This complement may be chosen arbitrarily, subject to the condition that $d_{1} \psi_{1}(1 \otimes z \otimes 1)=\psi_{0} \delta_{1}(1 \otimes z \otimes 1)$. Since $\psi_{0}, d_{1}, \delta_{1}$ all have degree 0 as maps, one may also choose $\psi_{1}$ to have degree 0 . In particular, note that $\psi_{1} \phi_{1}$ is the identity map on $X_{0,1}$. Now let $n \geq 2$ and assume that $\psi_{n-2}$ and $\psi_{n-1}$ have been defined to be degree-0 maps for which $d_{n-1} \psi_{n-1}=\psi_{n-2} \delta_{n-1}$ and $\psi_{n-1} \phi_{n-1}$ is the identity map on $X_{0, n-1}$. To define $\psi_{n}$, first note that $A^{\otimes(n+2)}$ contains the space $X_{0, n}$ as an $A^{e}$-submodule (see (2-14)) and the image of each $X_{i, j}$ under $\phi_{n}(n=i+j, i \geq 1)$. By construction, their images intersect in 0 , the image of $X_{0, n}$ under $\phi_{n}$ is free and, moreover, $\phi_{n}$ is injective on restriction to $X_{0, n}$. Choose a set of free generators of $\phi_{n}\left(X_{0, n}\right)$, and choose a set of free generators of its complement in $A^{\otimes(n+2)}$. For each chosen generator $x$ of $X_{0, n}$, we define $\psi_{n}\left(\phi_{n}(x)\right)$ to be $x$. On the complement of $\phi_{n}\left(X_{0, n}\right)$, define $\psi_{n}$ arbitrarily, subject to being a chain map of degree 0 . Thus, 
$\psi_{n} \phi_{n}$ is the identity map on $X_{0, n}$. Now for all $x \in X_{0, n}$, since $d_{n}(x) \in X_{0, n-1}$, we have that $\psi_{n-1} \phi_{n-1} d_{n}(x)=d_{n}(x)$, by induction. As $\delta_{n} \phi_{n}(x)=\phi_{n-1} d_{n}(x)$, it follows that $d_{n} \psi_{n} \phi_{n}(x)=\psi_{n-1} \delta_{n} \phi_{n}(x)$. So $\psi_{n}$ also extends the chain map from degree $n-1$ to degree $n$, as desired.

\section{Poincaré-Birkhoff-Witt theorem for Hopf algebra actions}

Consider the algebra $\mathscr{D}_{B, \kappa}$ from Notation 0.3 . The goal of this section is to prove our main result, Theorem 3.1. We provide necessary and sufficient conditions for $\mathscr{D}_{B, \kappa}$ to be a PBW deformation of $B \# H$ (Definition 0.1) as follows:

Theorem 3.1. The algebra $\mathscr{D}_{B, \kappa}$ is a $P B W$ deformation of $B \# H$ if and only if the following conditions hold:

(a) $\kappa$ is $H$-invariant (Definition 1.4);

If $\operatorname{dim}_{k} V \geq 3$, then the following equations hold for the maps $\kappa^{C} \otimes \mathrm{id}-\mathrm{id} \otimes \kappa^{C}$ and $\kappa^{L} \otimes \mathrm{id}-\mathrm{id} \otimes \kappa^{L}$, which are defined on the intersection $(I \otimes V) \cap(V \otimes I):$

(b) $\operatorname{Im}\left(\kappa^{L} \otimes \mathrm{id}-\mathrm{id} \otimes \kappa^{L}\right) \subseteq I$;

(c) $\kappa^{L} \circ\left(\kappa^{L} \otimes \mathrm{id}-\mathrm{id} \otimes \kappa^{L}\right)=-\left(\kappa^{C} \otimes \mathrm{id}-\mathrm{id} \otimes \kappa^{C}\right)$;

(d) $\kappa^{C} \circ\left(\mathrm{id} \otimes \kappa^{L}-\kappa^{L} \otimes \mathrm{id}\right) \equiv 0$.

Recall Notation 0.2: $B$ is generated by the $k$-vector space $V$ with quadratic relations $I \subset V \otimes V$, so $B=T_{k}(V) /(I)$. Moreover, consider:

Notation 3.2 $\left(U, T_{H}(U), R, P\right)$. Let $U:=V \otimes H$, which is an $H$-bimodule under the actions defined in Section 1A. Set $R=I \otimes H$, similarly an $H$-bimodule, and an $H$-subbimodule of $U \otimes_{H} U$. Let $P=\{r \otimes 1-\kappa(r) \mid r \in I\}$ be the relation space of $\mathscr{D}_{B, \kappa}$, generating an $H$-submodule of $H \oplus U \oplus\left(U \otimes_{H} U\right)$ in the tensor algebra

$$
T_{H}(U)=H \oplus U \oplus\left(U \otimes_{H} U\right) \oplus\left(U \otimes_{H} U \otimes_{H} U\right) \oplus \cdots .
$$

Note that $U_{H}^{\otimes_{H}^{n}} \cong V^{\otimes n} \otimes H$ as $k$-vector spaces. We see that $\pi(P)=R$, where the map $\pi$ is the projection onto the homogeneous quadratic part of $P$.

Consider the following preliminary results:

Lemma 3.3. Since $T_{H}(U)$ is canonically isomorphic to $T_{k}(V) \# H$, we have that

$$
T_{H}(U) /(P) \cong \mathscr{D}_{B, \kappa} \quad \text { and } \quad T_{H}(U) /(R) \cong\left(T_{k}(V) \# H\right) /(I) \cong B \# H,
$$

where (I) is identified with the ideal of $T_{k}(V) \# H$ generated by $I$.

Hence, $\mathscr{D}_{B, \kappa}$ is a PBW deformation of $B \# H$ if and only if $T_{H}(U) /(P)$ is a $P B W$ deformation of $T_{H}(U) /(R)$. 
Lemma 3.4 [Shepler and Witherspoon 2012b, Lemma 5.2]. If $T_{H}(U) /(P)$ is a $P B W$ deformation of $T_{H}(U) /(R)$, then the following conditions hold for maps $\alpha: R \rightarrow U$ and $\beta: R \rightarrow H$ for which $P=\{x-\alpha(x)-\beta(x) \mid x \in R\}:$

(i) $\operatorname{Im}\left(\alpha \otimes_{H}\right.$ id $\left.-\mathrm{id} \otimes_{H} \alpha\right) \subseteq R$.

(ii) $\alpha \circ\left(\alpha \otimes_{H}\right.$ id $\left.-\mathrm{id} \otimes_{H} \alpha\right)=-\left(\beta \otimes_{H}\right.$ id $\left.-\mathrm{id} \otimes_{H} \beta\right)$.

(iii) $\beta \circ\left(\right.$ id $\otimes_{H} \alpha-\alpha \otimes_{H}$ id $) \equiv 0$.

Here, the maps $\alpha \otimes_{H} \mathrm{id}-\mathrm{id} \otimes_{H} \alpha$ and $\beta \otimes_{H} \mathrm{id}-\mathrm{id} \otimes_{H} \beta$ are defined on the subspace $\left(R \otimes_{H} U\right) \cap\left(U \otimes_{H} R\right)$ of $T_{H}(U)$.

Remark 3.5. Given the maps $\kappa^{L}: I \rightarrow V \otimes H$ and $\kappa^{C}: I \rightarrow H$ as in Notation 0.2, we see that $\alpha=\kappa^{L} \otimes \mathrm{id}_{H}$ and $\beta=\kappa^{C} \otimes \mathrm{id}_{H}$.

Lemma 3.6. Consider the algebra

$$
\left(T_{H}(U) /(P)\right)_{t}:=\frac{T_{H}(U)[t]}{\left(x-\alpha(x) t-\beta(x) t^{2}\right)_{x \in R}} .
$$

We have that $\left(T_{H}(U) /(P)\right)_{t}$ is a PBW deformation of $T_{H}(U) /(R)$ over $k[t]$ if and only if $\mathscr{D}_{B, \kappa, t}$ (of Proposition 1.15) is a PBW deformation of $B \# H$ over $k[t]$.

Proof. This follows from Lemma 3.3 and Remark 3.5.

Now we provide the proof of Theorem 3.1. A somewhat shorter proof would suffice in case $H$ is semisimple: The first proof of [Shepler and Witherspoon 2012a, Theorem 3.1] may be generalized from semisimple group algebras to semisimple Hopf algebras. In that context, one has on hand a much smaller resolution than that which we will use below.

Proof of Theorem 3.1. Note that we will employ the identifications given in the lemmas and remark above, sometimes without comment. Namely, results from Section 2 will be used here where, for instance, $I$ is identified so that $R=I \otimes H$ and $B \# H$ is identified with $T_{H}(U) /(R)$.

Necessity of conditions (a)-(d). Let us first show that conditions (a)-(d) are necessary. Assume that $\mathscr{D}_{B, \kappa}$ is a PBW deformation of $B \# H$, and take $Q$ to be the relation space of $\mathscr{D}_{B, \kappa}$. Then, for all $h \in H$ and $r \in I$, we have that $h \cdot r-h \cdot(\kappa(r)) \in Q$. (Refer to Section 1A for the definition of these actions.) We also have that $h \cdot r-\kappa(h \cdot r) \in Q$, so $h \cdot(\kappa(r))-\kappa(h \cdot r) \in Q$. This implies that $h \cdot(\kappa(r))=\kappa(h \cdot r)$ in $\mathscr{D}_{B, \kappa}$, since $Q$ cannot contain nonzero elements in degree less than two. Thus, condition (a) holds. Moreover, by Lemma 3.3, $T_{H}(U) /(P)$ satisfies the PBW property.

Now by applying Lemma 3.4, we see that conditions (i), (ii), (iii) hold for $T_{H}(U) /(P)$. These conditions are equivalent to conditions (b), (c), (d) in Theorem 3.1 for the algebra $\mathscr{D}_{B, \kappa}$ by Notation 3.2 and Remark 3.5. Thus, if $\mathscr{D}_{B, \kappa}$ is a PBW deformation of $B \# H$, then conditions (a)-(d) of Theorem 3.1 must hold. 
Sufficiency of conditions (a)-(d). Conversely, let us assume that conditions (a)-(d) of Theorem 3.1 hold for the algebra $\mathscr{D}_{B, \kappa}$. Equivalently, by Notation 3.2, Lemma 3.3, and Remark 3.5, we assume the following for the algebra $T_{H}(U) /(P)$ :

- The maps $\alpha$ and $\beta$ are $H$-invariant.

- Conditions (i), (ii), (iii) of Lemma 3.4 hold.

The goal is to show that $\mathscr{D}_{B, \kappa}$ is a PBW deformation of $B \# H$, which, by Proposition 1.15 , is equivalent to showing that $\mathscr{D}_{B, \kappa, t}$ (of Proposition 1.15) is a graded deformation of $B \# H$ over $k[t]$. Hence, by Lemma 3.6, the goal is then equivalent to verifying that the algebra $\left(T_{H}(U) /(P)\right)_{t}$ is a graded deformation of $T_{H}(U) /(R)$ over $k[t]$. We thus have the following strategy:

- Let $A$ denote $T_{H}(U) /(R)$.

- Construct multiplication maps, $\mu_{i}: A \otimes A \rightarrow A$, as in Definition 1.5, subject to the restraints listed in Remark 1.8.

- Form the graded deformation $A_{t}$ of $A$ as in Definition 1.5.

- Conclude that $A^{\prime}:=T_{H}(U) /\left.(P) \cong\left(A_{t}\right)\right|_{t=1}$ is a PBW deformation of $A$ by Proposition 1.15.

We generalize the proof in [Shepler and Witherspoon 2012b] from group actions to Hopf algebra actions. Namely, we use the free resolution $X$. of the $A^{e}$-module $A$ in Construction 2.5 to define the maps $\mu_{i}$. Recall that $X$. is constructed from $C_{\bullet}=B_{\bullet}(A)$, the bar resolution of $H$, and from $D_{\bullet}$, the Koszul resolution of $B$.

Extending $\alpha$ and $\beta$ to be maps on $X$. We first extend $\alpha$ and $\beta$ to be maps on $X$. as follows. In degree $2, X_{2}$ contains as a direct summand $X_{0,2} \cong A \otimes I \otimes A$; see (2-14). As $\alpha, \beta$ are $H$-bilinear by $H$-invariance, we may extend them to $A^{e}$-module maps from $A \otimes R \otimes_{H} A \cong A \otimes I \otimes A$ to $A$ by composing with the multiplication map. By abuse of notation, denote these extended maps by $\alpha, \beta$ as well. Extend $\alpha$ and $\beta$ yet further by setting them equal to 0 on the summands $X_{2,0}$ and $X_{1,1}$ of $X_{2}$ so that they become maps $\alpha, \beta: X_{2} \rightarrow A$. More precisely, $\alpha, \beta \in \operatorname{Hom}_{A^{e}}\left(X_{2}, A\right) \cong \operatorname{Hom}_{k}\left(X_{2}^{\prime}, A\right)$ for $X_{2} \cong A \otimes X_{2}^{\prime} \otimes A$.

Construction of the multiplication map $\mu_{1}$. To build $\mu_{1} \in \operatorname{Hom}_{k}(A \otimes A, A) \cong$ $\operatorname{Hom}_{A^{e}}\left(A^{\otimes 4}, A\right)$, recall that it must be a Hochschild 2-cocycle as in (1-9). We will show that $\alpha: X_{2} \rightarrow A$ is a Hochschild 2-cocycle on $X_{\bullet}$, that is, $d_{3}^{*}(\alpha)=0$. Recall the chain maps of Lemma 2.13. We set $\mu_{1}=\psi_{2}^{*}(\alpha)$, which will be a Hochschild 2-cocycle on $B .(A)$, that is, $\delta_{3}^{*}\left(\mu_{1}\right)=0$.

To show that $d_{3}^{*}(\alpha): X_{3} \rightarrow A$ is the zero map, first note that $X_{3}=X_{0,3} \oplus$ $X_{1,2} \oplus X_{2,1} \oplus X_{3,0}$ from (2-9) and that the images of $X_{2,1}$ and $X_{3,0}$ under $d_{3}$ lie in $X_{1,1} \oplus X_{2,0}$. Since $\left.\alpha\right|_{X_{1,1} \oplus X_{2,0}} \equiv 0$ by the extension above, it suffices to show that $\left.d_{3}^{*}(\alpha)\right|_{X_{0,3}}$ and $\left.d_{3}^{*}(\alpha)\right|_{X_{1,2}}$ are zero maps. 
Rewriting condition (i) of Lemma 3.4, we see that it implies that $\alpha$ is 0 on the image of the differential on $X_{0,3}$ as follows: let $\sum_{i} 1 \otimes x_{i} \otimes y_{i} \otimes z_{i} \otimes 1 \in$ $A \otimes((I \otimes V) \cap(V \otimes I)) \otimes A=X_{0,3}$; see (2-14). Then

$$
\begin{aligned}
& \alpha\left(d_{3}\left(\sum_{i} 1 \otimes x_{i} \otimes y_{i} \otimes z_{i} \otimes 1\right)\right) \\
&=\alpha\left(\sum_{i} x_{i} \otimes y_{i} \otimes z_{i} \otimes 1-\sum_{i} 1 \otimes x_{i} y_{i} \otimes z_{i} \otimes 1\right. \\
& \quad\left.\quad+\sum_{i} 1 \otimes x_{i} \otimes y_{i} z_{i} \otimes 1-\sum_{i} 1 \otimes x_{i} \otimes y_{i} \otimes z_{i}\right) \\
&= \sum_{i}\left(x_{i} \alpha\left(y_{i} \otimes z_{i}\right)-\alpha\left(x_{i} \otimes y_{i}\right) z_{i}\right) .
\end{aligned}
$$

(To see this, note that applying the multiplication map of $A$ to elements in $I$ yields 0 .) Thus $d_{3}^{*}(\alpha)=\mathrm{id} \otimes \alpha-\alpha \otimes$ id on $X_{0,3}$; here, we identify id $\otimes \alpha-\alpha \otimes$ id with $m \circ$ (id $\otimes \alpha-\alpha \otimes \mathrm{id}$ ), where $m$ is the multiplication map on $A$. We see that condition (i) indeed implies (in fact, is equivalent to) $\left.d_{3}^{*}(\alpha)\right|_{X_{0,3}} \equiv 0$.

Next, we claim that $\alpha$ being $H$-invariant implies that $\alpha$ is also 0 on the image of the differential on $X_{1,2}$. Let $a, b \in A, h \in H$, and $r \in I$, and consider $a \otimes h \otimes r \otimes b$ as an element of $X_{1,2} \cong A \otimes H \otimes I \otimes A$ by Theorem 2.10(a). By the definition of the differential on $X_{1,2}$,

$$
\begin{aligned}
d(a \otimes h \otimes r \otimes b) & =d((a \otimes h \otimes 1) \otimes(1 \otimes r \otimes b)) \\
& =d(a \otimes h \otimes 1) \otimes(1 \otimes r \otimes b)-(a \otimes h \otimes 1) \otimes d(1 \otimes r \otimes b) .
\end{aligned}
$$

The second term lies in $X_{1,1}$, but $\alpha$ is 0 on $X_{1,1}$ by definition. Therefore,

$$
\begin{aligned}
\alpha(d(a \otimes h \otimes r \otimes b)) & =\alpha((a h \otimes 1-a \otimes h) \otimes(1 \otimes r \otimes b)) \\
& =\alpha\left(a h \otimes r \otimes b-\sum a \otimes\left(h_{1} \cdot r\right) \otimes h_{2} b\right) \\
& =a h \alpha(r) b-\sum a \alpha\left(h_{1} \cdot r\right) h_{2} b .
\end{aligned}
$$

Since $\alpha$ is $H$-invariant, we have that

$$
h \alpha(r)=\sum h_{1} \epsilon\left(h_{2}\right) \alpha(r)=\sum h_{1} \alpha(r) \epsilon\left(h_{2}\right)=\sum h_{1} \alpha(r) S\left(h_{2}\right) h_{3}=\sum \alpha\left(h_{1} \cdot r\right) h_{2},
$$

where the last equality used the fact that $\alpha$ is $H$-invariant. Thus, $\alpha$ is zero on the image of $d=d_{3}$ on $X_{1,2}$ by (3-7). It follows that $\alpha$ is a Hochschild 2-cocycle on $X$.

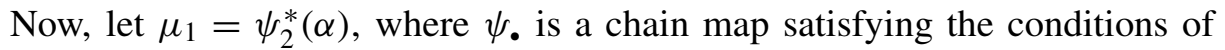
Lemma 2.13. We conclude that

$$
\delta_{3}^{*}\left(\mu_{1}\right)=\delta_{3}^{*}\left(\psi_{2}^{*}(\alpha)\right)=\psi_{3}^{*}\left(d_{3}^{*}(\alpha)\right) \equiv 0,
$$


as desired. So, we have a first-level graded deformation $A_{(1)}$ of $A$ with first multiplication map $\mu_{1}: A \otimes A \rightarrow A$.

As an aside, we also get that $\phi_{2}^{*}\left(\mu_{1}\right)=\alpha$ as cochains. To see this, first note that since $\alpha$ is homogeneous of degree -1 by its definition, so is $\mu_{1}$. Let $x \in X_{0,2}$. By Lemma 2.13, $\psi_{2} \phi_{2}(x)=x$, and thus

$$
\mu_{1} \phi_{2}(x)=\alpha \psi_{2} \phi_{2}(x)=\alpha(x) .
$$

Now let $y$ be a free generator of $X_{1,1}$ or of $X_{2,0}$, which may always be chosen to have degree 1 or 0 , respectively. Then $\psi_{2} \phi_{2}(y)$ has degree 1 or 0 respectively, implying that its component in $X_{0,2}$ is 0 . It follows that $\mu_{1} \phi_{2}(y)=\alpha \psi_{2} \phi_{2}(y)=0=\alpha(y)$; the last equation follows from the extension of $\alpha$ to $X$. Therefore $\phi_{2}^{*}\left(\mu_{1}\right)=\alpha$.

Construction of the multiplication map $\mu_{2}$. Given $\mu_{1}$ as above, note that the map $\mu_{2}$ must satisfy (1-10); that is, $\delta_{3}^{*}\left(\mu_{2}\right)=\mu_{1} \circ\left(\mu_{1} \otimes \mathrm{id}-\mathrm{id} \otimes \mu_{1}\right)$ as cochains on the bar resolution $\boldsymbol{B}$. $(A)$ of $A$. We will show that a modification of $\psi_{2}^{*}(\beta)$ is such a map, as follows:

First, note that $\beta=\phi_{2}^{*}\left(\psi_{2}^{*}(\beta)\right)$ as cochains, by an argument similar to that above for $\alpha$. Moreover, condition (ii) implies that $d_{3}^{*}(\beta)=\alpha \circ\left(\alpha \otimes_{H} \mathrm{id}-\mathrm{id} \otimes_{H} \alpha\right)$ as cochains on $X_{0,3}$. Let

$$
\gamma=\delta_{3}^{*} \psi_{2}^{*}(\beta)-\mu_{1} \circ\left(\mu_{1} \otimes \mathrm{id}-\mathrm{id} \otimes \mu_{1}\right)
$$

Then $\phi_{3}^{*}(\gamma)$ is zero on $X_{0,3}: \phi_{3}^{*} \delta_{3}^{*} \psi_{2}^{*}(\beta)=d_{3}^{*}(\beta)$ and $\phi_{3}^{*}\left(\mu_{1} \circ\left(\mu_{1} \otimes \mathrm{id}-\mathrm{id} \otimes \mu_{1}\right)\right)=$ $\alpha \circ(\alpha \otimes \mathrm{id}-\mathrm{id} \otimes \alpha)$ by Lemma 3.4(ii). To see the last statement, note that the image of $\phi_{3}$ on $X_{0,3}$ is contained in $A \otimes((I \otimes V) \cap(V \otimes I)) \otimes A$ with $\phi^{*}\left(\mu_{1}\right)=\alpha$. We also see that $\phi_{3}^{*}(\gamma)$ is 0 on $X_{2,1}$ and $X_{3,0}$ since it is a map of degree -2 . We claim it is also 0 on $X_{1,2}$ as follows. As an $A^{e}$-module, the image of $X_{1,2}$ under $\phi_{3}$ is generated by elements of degree 2 . Since $\mu_{1}=\psi_{2}^{*}(\alpha)$, it is zero on elements of degree less than two, and so the map $\mu_{1} \circ\left(\mu_{1} \otimes \mathrm{id}-\mathrm{id} \otimes \mu_{1}\right)$ must be 0 on the image of $X_{1,2}$ under $\phi_{3}$. Since $\beta$ is $H$-invariant, and thus $\beta$ is a cocycle (see the argument following (3-7)) and $\phi_{2}^{*} \psi_{2}^{*}(\beta)=\beta$, we have that $\phi_{3}^{*} \delta_{3}^{*} \psi_{2}^{*}(\beta)=d_{3}^{*} \phi_{2}^{*} \psi_{2}^{*}(\beta)=d_{3}^{*}(\beta)$ is 0 on $X_{1,2}$. Therefore $\phi_{3}^{*}(\gamma)$ is 0 on $X_{1,2}$.

We have shown that $\phi_{3}^{*}(\gamma)$ is 0 on all of $X_{3}$, and so $\gamma$ must be a coboundary on the bar resolution $\boldsymbol{B}$. $(A)$ of $A$. Thus there is a 2-cochain $\mu$ of degree -2 on the bar resolution with

$$
\delta_{3}^{*}(\mu)=\gamma .
$$

Consider $\psi_{2}^{*}(\beta)-\mu$, yet note that $\phi_{2}^{*}\left(\psi_{2}^{*}(\beta)-\mu\right)$ may not agree with $\beta$ on $X_{2}$. We need such a statement for the next step of constructing $\mu_{3}$. Now,

$$
d_{3}^{*} \phi_{2}^{*}(\mu)=\phi_{3}^{*} \delta_{3}^{*}(\mu)=\phi_{3}^{*}(\gamma)=0
$$


so the 2-cochain $\phi_{2}^{*}(\mu)$ is a cocycle on the complex $X$. Thus, $\phi_{2}^{*}(\mu)$ lifts to a cocycle $\mu^{\prime}$ of degree -2 on the bar complex $\boldsymbol{B}$. $(A)$. In other words, $\phi_{2}^{*}\left(\psi_{2}^{*}(\beta)-\mu+\mu^{\prime}\right)$ agrees with $\beta$ on $X_{2}$.

Moreover, $\delta_{3}^{*}\left(\mu^{\prime}\right)=0$, and by (3-8) and (3-9), we have that $\delta_{3}^{*}\left(\psi_{2}^{*}(\beta)-\mu\right)=$ $\mu_{1} \circ\left(\mu_{1} \otimes \mathrm{id}-\mathrm{id} \otimes \mu_{1}\right)$. So,

$$
\delta_{3}^{*}\left(\psi_{2}^{*}(\beta)-\mu+\mu^{\prime}\right)-\mu_{1} \circ\left(\mu_{1} \otimes \mathrm{id}-\mathrm{id} \otimes \mu_{1}\right)=0
$$

on the bar resolution $\boldsymbol{B}$. $(A)$ of $A$.

Thus, setting $\mu_{2}$ equal to $\psi_{2}^{*}(\beta)-\mu+\mu^{\prime}$, we have maps $\mu_{1}, \mu_{2}$ to obtain a second-level graded deformation $A_{(2)}$ of $A$ extending $A_{(1)}$.

Construction of the multiplication map $\mu_{3}$. Recall the restraint on $\mu_{3}$ given in (1-11): $\mu_{3}$ is a cochain on $\boldsymbol{B} .(A)$ whose coboundary is given by $\mu_{1} \circ\left(\mu_{2} \otimes \mathrm{id}-\mathrm{id} \otimes \mu_{2}\right)+$ $\mu_{2} \circ\left(\mu_{1} \otimes \mathrm{id}-\mathrm{id} \otimes \mu_{1}\right)$. We construct $\mu_{3}$ as follows.

By (3-10) and condition (iii) of Lemma 3.4, we have that $\mu_{2} \circ\left(\mu_{1} \otimes \mathrm{id}-\mathrm{id} \otimes \mu_{1}\right)$ is 0 on the image of $\phi$. By degree considerations, $\mu_{1} \circ\left(\mu_{2} \otimes \mathrm{id}-\mathrm{id} \otimes \mu_{2}\right)$ is always 0 on the image of $\phi$. Therefore, the obstruction

$$
\mu_{2} \circ\left(\mu_{1} \otimes \mathrm{id}-\mathrm{id} \otimes \mu_{1}\right)+\mu_{1} \circ\left(\mu_{2} \otimes \mathrm{id}-\mathrm{id} \otimes \mu_{2}\right)
$$

is a coboundary. Thus there exists a 2 -cochain $\mu_{3}$, necessarily having degree -3 , satisfying the restraint given above, and the deformation lifts to the third level.

Construction of the multiplication maps $\mu_{i}$ for $i \geq 4$. The obstruction for a thirdlevel graded deformation $A_{(3)}$ of $A$ to lift to the fourth level lies in $\mathrm{HH}^{3,-4}(A)$ by Proposition 1.13. We apply $\phi_{3}^{*}$ to this obstruction to obtain a cochain on $X_{3}$. Since there are no cochains of degree -4 on $X_{3}$ by definition (as it is generated by elements of degree 3 or less), $\phi_{3}^{*}$ applied to the obstruction is automatically zero. Therefore, the deformation may be continued to the fourth level. Similar arguments show that it can be continued to the fifth level, and so on.

Construction of $A_{t}$. Let $A_{t}$ be the graded deformation of $A$ that we obtain in this manner (Definition 1.5). Then, $A_{t}$ is the $k$-vector space $A[t]$ with multiplication defined for all $a_{1}, a_{2} \in A$ by

$$
a_{1} * a_{2}=a_{1} a_{2}+\mu_{1}\left(a_{1} \otimes a_{2}\right) t+\mu_{2}\left(a_{1} \otimes a_{2}\right) t^{2}+\mu_{3}\left(a_{1} \otimes a_{2}\right) t^{3}+\cdots,
$$

where $a_{1} a_{2}$ is the product in $A$ and each $\mu_{i}: A \otimes A \rightarrow A$ is a $k$-linear map of homogeneous degree $-i$. Now for any $r$ in $R, \mu_{1}(r)=\left(\psi_{2}^{*} \alpha\right)(r)$ and $\mu_{2}(r)=$ $\left(\psi_{2}^{*}(\beta)-\mu+\mu^{\prime}\right)(r)$ by construction, and $\mu_{i}(r)=0$ for $i \geq 3$ since $\operatorname{deg}(r)=2$.

Conclusion that $A^{\prime}:=T_{H}(U) /(P)$ is a $P B W$ deformation of $A=T_{H}(U) /(R)$. Now we show that $A^{\prime}:=T_{H}(U) /(P)$ is isomorphic, as a filtered algebra, to the fiber of the deformation $A_{t}$ at $t=1$ as follows. Let $A^{\prime \prime}=\left.\left(A_{t}\right)\right|_{t=1}$. Then $A^{\prime \prime}$ is generated by $V$ and $H$ and one thus obtains a surjective algebra homomorphism 
$T_{H}(U) \cong T_{k}(V) \# H \rightarrow A^{\prime \prime}$. The elements of $P$ lie in the kernel (by definition of $\left.A^{\prime \prime}\right)$, and thus this map induces a surjective algebra homomorphism $A^{\prime}=T_{H}(U) /(P) \rightarrow$ $A^{\prime \prime}$. This map is in fact an isomorphism of filtered algebras by a dimension argument in each degree. Therefore $A^{\prime}$ is a PBW deformation of $A$, since $A^{\prime \prime}$ is one (Proposition 1.14).

\section{Examples}

For our examples, we restrict $k$ to be an algebraically closed field of characteristic zero. There are many interesting examples, both known and new, in this setting. Less is known about Hopf actions on Koszul algebras and corresponding deformations in positive characteristic.

As an application of Theorem 3.1, we provide various examples of PBW deformations $\mathscr{D}_{B, \kappa}$ of smash products $B \# H$; recall Notations 0.2 and 0.3 . We do this by describing deformation parameter(s) $\kappa=\kappa^{C}+\kappa^{L}$ below. In particular, Examples 4.1, 4.2, and 4.4 involve semisimple Hopf actions, and Examples 4.13, 4.16, and 4.18 involve nonsemisimple Hopf actions on (skew) polynomial rings. Recall that skew polynomial rings are Koszul by [Polishchuk and Positselski 2005, Example 4.2.1 and Theorem 4.3.1].

4A. Semisimple Hopf actions. We begin by revisiting the well-known PBW deformations of Crawley-Boevey and Holland [1998].

Example 4.1. Take $H=k \Gamma$, for $\Gamma$ a finite subgroup of $S L_{2}(k)$, and $B=k[u, v]$. For $g=\left(\begin{array}{ll}a & c \\ b & d\end{array}\right) \in \Gamma$, let the action of $g$ on $B$ be given by $g \cdot u=a u+c v$ and $g \cdot v=b u+d v$.

By [Crawley-Boevey and Holland 1998], the deformation parameter $\kappa$ of the PBW deformation $\mathscr{D}_{B, \kappa}$ of $B \# H$ must be in the center of $\Gamma$, which we verify again with Theorem 3.1. We assume here that $\kappa^{L} \equiv 0$, as in that work.

Since $\operatorname{dim}_{k} V=2$, only condition (a) of Theorem 3.1 applies. So we have for all $g \in \Gamma$ that $g \cdot(\kappa(u v-v u))=\kappa(g \cdot(u v-v u))$. Now since the determinant of $g$ is 1 , $g \cdot(\kappa(u v-v u))=\kappa(u v-v u)$, and the image of $\kappa$ lies in the center of $k \Gamma$. That is,

$$
\mathscr{D}_{B, \kappa}=\frac{k\langle u, v\rangle \# k \Gamma}{(u v-v u-\lambda)}
$$

is a PBW deformation of $k[u, v] \# k \Gamma$ if and only if $\lambda \in Z(k \Gamma)$.

It is worth pointing out that there are analogues of Crawley-Boevey-Holland algebras when working in positive characteristic; see the work of Emily Norton [2013] for some examples that are quite different from those in characteristic zero.

The following two Hopf actions were produced by Walton in joint work with Kenneth Chan, Ellen Kirkman, and James Zhang [Chan et al. 2012]. We thank them for permitting us to use these results. 
Example 4.2. Let $H:=H_{8}$ be the unique noncommutative noncocommutative semisimple 8-dimensional Hopf algebra [Kac and Paljutkin 1966; Masuoka 1995], and let $B=k\langle u, v\rangle /\left(u^{2}+v^{2}\right)$ (which is isomorphic to the skew polynomial ring $k\langle u, v\rangle /(u v+v u))$. The Hopf algebra $H_{8}$ is generated by $x, y, z$, and the relations are

$$
x^{2}=y^{2}=1, \quad x y=y x, \quad z x=y z, \quad z y=x z, \quad z^{2}=\frac{1}{2}(1+x+y-x y) .
$$

The rest of the structure of $H_{8}$ and the left $H_{8}$-action on $B$ are given by $\Delta(x)=x \otimes x, \quad \Delta(y)=y \otimes y, \quad \Delta(z)=\frac{1}{2}(1 \otimes 1+1 \otimes x+y \otimes 1-y \otimes x)(z \otimes z)$,

$$
\begin{gathered}
\epsilon(x)=\epsilon(y)=\epsilon(z)=1, \quad S(x)=x, \quad S(y)=y, \quad S(z)=z, \\
x \cdot u=-u, \quad x \cdot v=v, \quad y \cdot u=u, \quad y \cdot v=-v, \quad z \cdot u=v, \quad z \cdot v=u .
\end{gathered}
$$

Let $r:=u^{2}+v^{2}$ and note that

$$
x \cdot r=r, \quad y \cdot r=r, \quad z \cdot r=r,
$$

so the ideal of relations of $B, I=\langle r\rangle$, is $H$-stable.

Since $\operatorname{dim}_{k} V=2$, only condition (a) of Theorem 3.1 applies. We begin by computing $\kappa^{C}$. Let

$$
\kappa^{C}(r)=\gamma_{0}+\gamma_{1} x+\gamma_{2} y+\gamma_{3} x y+\gamma_{4} z+\gamma_{5} x z+\gamma_{6} y z+\gamma_{7} x y z
$$

for some scalars $\gamma_{i} \in k$. Since $h \cdot\left(\kappa^{C}(r)\right)=\sum h_{1}\left(\kappa^{C}(r)\right) S\left(h_{2}\right)$ (see Section 1A), both $x \cdot\left(\kappa^{C}(r)\right)=\kappa^{C}(r)$ and $y \cdot\left(\kappa^{C}(r)\right)=\kappa^{C}(r)$ imply that $\gamma_{7}=\gamma_{4}$ and $\gamma_{6}=\gamma_{5}$. Moreover,

$$
z \cdot\left(\kappa^{C}(r)\right)=\gamma_{0}+\gamma_{2} x+\gamma_{1} y+\gamma_{3} x y+\gamma_{4} z+\gamma_{5} x z+\gamma_{5} y z+\gamma_{4} x y z=\kappa^{C}(r),
$$

which implies that $\gamma_{2}=\gamma_{1}$. Thus,

$$
\begin{aligned}
\kappa^{C}(r) & =: g\left(\gamma_{0}, \gamma_{1}, \gamma_{3}, \gamma_{4}, \gamma_{5}\right) \\
& =\gamma_{0}+\gamma_{1}(x+y)+\gamma_{3} x y+\gamma_{4}(z+x y z)+\gamma_{5}(x z+y z) .
\end{aligned}
$$

On the other hand, let $\kappa^{L}(r)=u \otimes f+v \otimes f^{\prime} \in V \otimes H$ with

$$
\begin{aligned}
& f=\delta_{0}+\delta_{1} x+\delta_{2} y+\delta_{3} x y+\delta_{4} z+\delta_{5} x z+\delta_{6} y z+\delta_{7} x y z, \\
& f^{\prime}=\delta_{0}^{\prime}+\delta_{1}^{\prime} x+\delta_{2}^{\prime} y+\delta_{3}^{\prime} x y+\delta_{4}^{\prime} z+\delta_{5}^{\prime} x z+\delta_{6}^{\prime} y z+\delta_{7}^{\prime} x y z,
\end{aligned}
$$

for some scalars $\delta_{i}, \delta_{i}^{\prime} \in k$. Note that $h \cdot\left(\kappa^{L}(r)\right)=\sum h_{1} \cdot u \otimes h_{2} f S\left(h_{3}\right)+$ $\sum h_{1} \cdot v \otimes h_{2} f^{\prime} S\left(h_{3}\right)$ (see Section 1A). Since $x \cdot\left(\kappa^{L}(r)\right)=\kappa^{L}(r)$, it follows that:

$$
\delta_{0}=\delta_{1}=\delta_{2}=\delta_{3}=0, \quad \delta_{4}=-\delta_{7}, \quad \delta_{5}=-\delta_{6} \quad \delta_{4}^{\prime}=\delta_{7}^{\prime}, \quad \text { and } \quad \delta_{5}^{\prime}=\delta_{6}^{\prime} .
$$

By considering the coefficient of $u$ in the equation $y \cdot\left(\kappa^{L}(r)\right)=\kappa^{L}(r)$, we now find that $f=0$. Similarly, by considering the coefficient of $v$ in the equation $y \cdot\left(\kappa^{L}(r)\right)=\kappa^{L}(r)$, we find that $f^{\prime}=0$. Hence, $\kappa^{L}(r)=0$. 
Thus the deformation parameter $\kappa$ of $\mathscr{D}_{B, \kappa}$ equals its constant part $\kappa^{C}$, which depends on five scalar parameters as described above. In short,

$$
\mathscr{D}_{B, \kappa}=\frac{k\langle u, v\rangle \# H_{8}}{\left(u^{2}+v^{2}-\kappa\left(u^{2}+v^{2}\right)\right)}
$$

is a PBW deformation of $\left(k\langle u, v\rangle /\left(u^{2}+v^{2}\right)\right) \# H_{8}$ if and only if $\kappa\left(u^{2}+v^{2}\right)=$ $g\left(\gamma_{0}, \gamma_{1}, \gamma_{3}, \gamma_{4}, \gamma_{5}\right)$ as given in (4-3). This yields a five-parameter family of PBW deformations of $B \# H_{8}$.

Example 4.4. Let $H$ be $H_{a: 1}$, one of the 16-dimensional semisimple Hopf algebras classified in [Kashina 2000], and let $B$ be the skew polynomial ring

$$
B=\frac{k\langle t, u, v, w\rangle}{\left(\begin{array}{lll}
r_{t u}:=t u-u t, & r_{t v}:=t v-v t, & r_{t w}:=t w+w t \\
r_{u v}:=u v-v u, & r_{u w}:=u w+w u, & r_{v w}:=v w-w v
\end{array}\right)} .
$$

The Hopf algebra $H_{a: 1}$ is generated by $x, y, z$, subject to the relations

$$
x^{4}=y^{2}=z^{2}=1, \quad y x=x y, \quad z x=x y z, \quad z y=y z .
$$

The rest of the structure of $H_{a: 1}$ and the left $H_{a: 1}$-action on $B$ are given by

$$
\begin{gathered}
\Delta(x)=x \otimes x, \quad \Delta(y)=y \otimes y, \quad \Delta(z)=\frac{1}{2}\left(1 \otimes 1+1 \otimes x^{2}+y \otimes 1-y \otimes x^{2}\right)(z \otimes z), \\
\epsilon(x)=\epsilon(y)=\epsilon(z)=1, \quad S(x)=x^{3}, \quad S(y)=y, \quad S(z)=\frac{1}{2}\left(1+x^{2}+y-x^{2} y\right) z, \\
x \cdot t=i t, \quad y \cdot t=-t, \quad z \cdot t=u, \quad x \cdot u=-i u, \quad y \cdot u=-u, \quad z \cdot u=t, \\
x \cdot v=v, \quad y \cdot v=-v, \quad z \cdot v=w, \quad x \cdot w=-w, \quad y \cdot w=-w, \quad z \cdot w=v,
\end{gathered}
$$

where $i$ is a primitive fourth root of unity in $k$. Note that

$$
\begin{aligned}
& x \cdot r_{t u}=r_{t u}, \quad x \cdot r_{t v}=i r_{t v}, \quad x \cdot r_{t w}=-i r_{t w}, \\
& x \cdot r_{u v}=-i r_{u v}, \quad x \cdot r_{u w}=i r_{u w}, \quad x \cdot r_{v w}=-r_{v w} \text {, } \\
& y \cdot r_{t u}=r_{t u}, \quad y \cdot r_{t v}=r_{t v}, \quad y \cdot r_{t w}=r_{t w} \text {, } \\
& y \cdot r_{u v}=r_{u v}, \quad y \cdot r_{u w}=r_{u w}, \quad y \cdot r_{v w}=r_{v w} \text {, } \\
& z \cdot r_{t u}=r_{t u}, \quad z \cdot r_{t v}=r_{u w}, \quad z \cdot r_{t w}=r_{u v}, \\
& z \cdot r_{u v}=r_{t w}, \quad z \cdot r_{u w}=r_{t v}, \quad z \cdot r_{v w}=-r_{v w} \text {. }
\end{aligned}
$$

So, the ideal of relations $I=\left\langle r_{t u}, r_{t v}, r_{t w}, r_{u v}, r_{u w}, r_{v w}\right\rangle$ of $B$ is $H$-stable.

Now we compute the possible values $\kappa^{C}(r) \in H$ for all $r \in I$, under condition (a) of Theorem 3.1. Take $\kappa^{C}(r)=g(\underline{\gamma}) \in H$ given by

$$
\begin{aligned}
g(\underline{\gamma})= & \gamma_{0}+\gamma_{1} x+\gamma_{2} x^{2}+\gamma_{3} x^{3}+\gamma_{4} y+\gamma_{5} x y+\gamma_{6} x^{2} y+\gamma_{7} x^{3} y \\
& +\gamma_{8} z+\gamma_{9} x z+\gamma_{10} x^{2} z+\gamma_{11} x^{3} z+\gamma_{12} y z+\gamma_{13} x y z+\gamma_{14} x^{2} y z+\gamma_{15} x^{3} y z,
\end{aligned}
$$


where $\gamma_{i} \in k$. Note that $h \cdot g(\underline{\gamma})=\sum h_{1} g(\underline{\gamma}) S\left(h_{2}\right)$. With the assistance of Affine, a subpackage of Maxima, we have the following computations:

$$
\begin{aligned}
x \cdot g(\underline{\gamma})=x g(\underline{\gamma}) x^{3}=\gamma_{0} & +\gamma_{1} x+\gamma_{2} x^{2}+\gamma_{3} x^{3}+\gamma_{4} y+\gamma_{5} x y \\
+ & \gamma_{6} x^{2} y+\gamma_{7} x^{3} y+\gamma_{8} y z+\gamma_{9} x y z+\gamma_{10} x^{2} y z \\
& +\gamma_{11} x^{3} y z+\gamma_{12} z+\gamma_{13} x z+\gamma_{14} x^{2} z+\gamma_{15} x^{3} z ;
\end{aligned}
$$

(4-6) $y \cdot g(\underline{\gamma})=y g(\underline{\gamma}) y=g(\underline{\gamma})$;

$$
\begin{gathered}
z \cdot g(\underline{\gamma})=\frac{1}{2}\left(z g(\underline{\gamma}) S(z)+z g(\underline{\gamma}) S\left(x^{2} z\right)+y z g(\underline{\gamma}) S(z)-y z g(\underline{\gamma}) S\left(x^{2} z\right)\right) \\
=\gamma_{0}+\gamma_{1} x y+\gamma_{2} x^{2}+\gamma_{3} x^{3} y+\gamma_{4} y+\gamma_{5} x \\
\quad+\gamma_{6} x^{2} y+\gamma_{7} x^{3}+\gamma_{8} z+\gamma_{9} x y z+\gamma_{10} x^{2} z \\
\quad+\gamma_{11} x^{3} y z+\gamma_{12} y z+\gamma_{13} x z+\gamma_{14} x^{2} y z+\gamma_{15} x^{3} z .
\end{gathered}
$$

For $r_{t u}$, let $\kappa^{C}\left(r_{t u}\right)=g(\underline{\gamma})$. We have that $x \cdot \kappa^{C}\left(r_{t u}\right)=\kappa^{C}\left(r_{t u}\right)$ and $y \cdot \kappa^{C}\left(r_{t u}\right)=$ $\kappa^{C}\left(r_{t u}\right)$ imply that $\gamma_{8}=\gamma_{12}, \gamma_{9}=\gamma_{13}, \gamma_{10}=\gamma_{14}, \gamma_{11}=\gamma_{15}$. Moreover, $z \cdot \kappa^{C}\left(r_{t u}\right)=$ $\kappa^{C}\left(r_{t u}\right)$ implies that $\gamma_{1}=\gamma_{5}, \gamma_{3}=\gamma_{7}, \gamma_{9}=\gamma_{13}, \gamma_{11}=\gamma_{15}$. Therefore,

$$
\begin{aligned}
\kappa^{C}\left(r_{t u}\right)= & g\left(\gamma_{0}, \gamma_{1}, \gamma_{2}, \gamma_{3}, \gamma_{4}, \gamma_{6}, \gamma_{8}, \gamma_{9}, \gamma_{10}, \gamma_{11}\right) \\
= & \gamma_{0}+\gamma_{1}(x+x y)+\gamma_{2} x^{2}+\gamma_{3}\left(x^{3}+x^{3} y\right)+\gamma_{4} y+\gamma_{6} x^{2} y \\
& +\gamma_{8}(z+y z)+\gamma_{9}(x z+x y z)+\gamma_{10}\left(x^{2} z+x^{2} y z\right)+\gamma_{11}\left(x^{3} z+x^{3} y z\right) .
\end{aligned}
$$

For $r_{v w}$, let $\kappa^{C}\left(r_{v w}\right)=g\left(\underline{\gamma}^{\prime}\right)$. We have that $x \cdot \kappa^{C}\left(r_{v w}\right)=-\kappa^{C}\left(r_{v w}\right)$ and $y \cdot \kappa^{C}\left(r_{v w}\right)=\kappa^{C}\left(r_{v w}\right)$ implies that $\gamma_{0}^{\prime}=\cdots=\gamma_{7}^{\prime}=0, \gamma_{8}^{\prime}=-\gamma_{12}^{\prime}, \gamma_{9}^{\prime}=-\gamma_{13}^{\prime}$, $\gamma_{10}^{\prime}=-\gamma_{14}^{\prime}$, and $\gamma_{11}^{\prime}=-\gamma_{15}^{\prime}$. Moreover, we have that $z \cdot \kappa^{C}\left(r_{v w}\right)=-\kappa^{C}\left(r_{v w}\right)$. So the conditions on $\gamma_{i}^{\prime}$ in (4-7) then imply that $\gamma_{i}^{\prime}=0$ for $i=0, \ldots, 7,8,10,12,14$ with $\gamma_{9}^{\prime}=-\gamma_{13}^{\prime}, \gamma_{11}^{\prime}=-\gamma_{15}^{\prime}$. Thus,

$$
\kappa^{C}\left(r_{v w}\right)=g\left(\gamma_{9}^{\prime}, \gamma_{11}^{\prime}\right)=\gamma_{9}^{\prime}(x z-x y z)+\gamma_{11}^{\prime}\left(x^{3} z-x^{3} y z\right) .
$$

For $r \neq r_{t u}, r_{v w}$, we have that $x \cdot \kappa^{C}(r)= \pm i \kappa^{C}(r)$ implies that $\kappa^{C}(r)=0$.

We compute $\kappa^{L}(r)$ under condition (a) of Theorem 3.1. Fix $r \in I$ and let

$$
\kappa^{L}(r)=t \otimes f_{t}+u \otimes f_{u}+v \otimes f_{v}+w \otimes f_{w} \in V \otimes H
$$

for some $f_{t}, f_{u}, f_{v}, f_{w} \in H$. Since $y$ is central in $H$ and $y \cdot r=r$ for each relation $r$, we have that

$$
\begin{aligned}
\kappa^{L}(r) & =y \cdot \kappa^{L}(r) \\
& =y \cdot t \otimes y f_{t} S(y)+y \cdot u \otimes y f_{u} S(y)+y \cdot v \otimes y f_{v} S(y)+y \cdot w \otimes y f_{w} S(y) \\
& =-t \otimes f_{t}-u \otimes f_{u}-v \otimes f_{v}-w \otimes f_{w}=-\kappa^{L}(r) .
\end{aligned}
$$

Thus, $\kappa^{L}(r)=0$. 
To finish, we apply to $\kappa$ conditions (b)-(d) of Theorem 3.1. Since $\kappa^{L}(r)=0$ for all $r \in I$, only condition (c) is pertinent. Namely, we only need to impose $\kappa^{C} \otimes \mathrm{id}=\mathrm{id} \otimes \kappa^{C}$ as maps on $(I \otimes V) \cap(V \otimes I)$. This intersection is a 4-dimensional $k$-vector space with basis

$$
\begin{aligned}
& s_{t u v}:=t u v-t v u-u t v+u v t+v t u-v u t, \\
& s_{t u w}:=t u w+t w u-u t w-u w t+w t u-w u t, \\
& s_{t v w}:=t v w-t w v-v t w+v w t+w t v-w v t, \\
& s_{u v w}:=u v w-u w v-v u w-v w u-w u v+w v u .
\end{aligned}
$$

Since $\kappa^{C}\left(r_{t v}\right)=\kappa^{C}\left(r_{u v}\right)=0$, we get that

$$
\begin{aligned}
\left(\kappa^{C} \otimes \mathrm{id}-\mathrm{id} \otimes \kappa^{C}\right)\left(s_{t u v}\right)= & \kappa^{C}\left(r_{u v}\right) t-\kappa^{C}\left(r_{t v}\right) u+\kappa^{C}\left(r_{t u}\right) v \\
& -t \kappa^{C}\left(r_{u v}\right)+u \kappa^{C}\left(r_{t v}\right)-v \kappa^{C}\left(r_{t u}\right) \\
= & \kappa^{C}\left(r_{t u}\right) v-v \kappa^{C}\left(r_{t u}\right) .
\end{aligned}
$$

Identify $b \in V$ with $b \# 1 \in A$ and $h \in H$ with $1 \# h \in A$. Recall that in $A$ we have $(1 \# h)(b \# 1)=\sum\left(h_{1} \cdot b\right) \# h_{2}$. Now by using (4-8) and by setting (4-10) equal to 0 , we get that

$$
\kappa^{C}\left(r_{t u}\right)=g\left(\gamma_{0}, \gamma_{2}\right)=\gamma_{0}+\gamma_{2} x^{2} .
$$

Moreover,

$$
\begin{aligned}
\left(\kappa^{C} \otimes \mathrm{id}-\mathrm{id} \otimes \kappa^{C}\right)\left(s_{t u w}\right)= & -\kappa^{C}\left(r_{u w}\right) t+\kappa^{C}\left(r_{t w}\right) u+\kappa^{C}\left(r_{t u}\right) w \\
& -t \kappa^{C}\left(r_{u w}\right)+u \kappa^{C}\left(r_{t w}\right)-w \kappa^{C}\left(r_{t u}\right) \\
= & \kappa^{C}\left(r_{t u}\right) w-w \kappa^{C}\left(r_{t u}\right)=0
\end{aligned}
$$

imposes no new restrictions on $\kappa^{C}\left(r_{t u}\right)$, nor do $\left(\kappa^{C} \otimes \mathrm{id}-\mathrm{id} \otimes \kappa^{C}\right)\left(s_{t v w}\right)=0$, $\left(\kappa^{C} \otimes \mathrm{id}-\mathrm{id} \otimes \kappa^{C}\right)\left(s_{u v w}\right)=0$. Therefore, $\kappa^{C}\left(r_{t u}\right)$ is given by (4-11).

To compute $\kappa^{C}\left(r_{v w}\right)$, consider the calculation

$$
\begin{aligned}
\left(\kappa^{C} \otimes \mathrm{id}-\mathrm{id} \otimes \kappa^{C}\right)\left(s_{t v w}\right)= & \kappa^{C}\left(r_{v w}\right) t-\kappa^{C}\left(r_{t w}\right) v+\kappa^{C}\left(r_{t v}\right) w \\
& -t \kappa^{C}\left(r_{v w}\right)+v \kappa^{C}\left(r_{t w}\right)-w \kappa^{C}\left(r_{t v}\right) \\
= & \kappa^{C}\left(r_{v w}\right) t-t \kappa^{C}\left(r_{v w}\right) .
\end{aligned}
$$

Now by using (4-9) and by setting (4-12) equal to 0 , we get that $\kappa^{C}\left(r_{v w}\right)=0$.

Therefore, the filtered algebra $\mathscr{D}_{B, \kappa}$ is a PBW deformation of $B \# H_{a: 1}$ if and only if the deformation parameter $\kappa=\kappa^{C}$ of $\mathscr{D}_{B, \kappa}$ is defined by (4-11) for the relation $r_{t u}$, and $\kappa^{C}(r)=0$ for $r \neq r_{t u}$. Hence, we have a two-parameter family of PBW deformations of $B \# H_{a: 1}$. 
4B. Nonsemisimple Hopf actions. Here, we consider nonsemisimple Hopf actions to illustrate Theorem 3.1. We begin with an example of a Taft algebra action.

Example 4.13. Let $H=T(n)$, the $n^{2}$-dimensional nonsemisimple Taft algebra. We take $n \geq 3$ here and we consider the (slightly different) case $n=2$ (the Sweedler algebra) in Example 4.16 below. Let $B=k[u, v]$. The Hopf algebra $T(n)$ is generated by $g, x$ and the relations are $g^{n}=1, x^{n}=0$, and $x g=\zeta g x$, for $\zeta \in k^{\times}$ a primitive $n$-th root of unity. The rest of the structure of $T(n)$ and the left $T(n)$ action on $B$ are given by

$$
\begin{gathered}
\Delta(g)=g \otimes g, \quad \Delta(x)=g \otimes x+x \otimes 1, \quad \epsilon(g)=1, \quad \epsilon(x)=0, \\
\quad S(g)=g^{-1}=g^{n-1}, \quad S(x)=-g^{n-1} x, \\
g \cdot u=u, \quad g \cdot v=\zeta^{-1} v, \quad x \cdot u=0, \quad x \cdot v=u .
\end{gathered}
$$

Let $r:=u v-v u$ and note that $g \cdot r=\zeta^{-1} r$ and $x \cdot r=0$. Hence, the ideal of relations $I=\langle r\rangle$ of $B$ is $H$-stable.

Since $\operatorname{dim}_{k} V=2$, only condition (a) of Theorem 3.1 applies. Now, we compute $\kappa^{C}$. Let $\kappa^{C}(r)=\sum_{i, j=0}^{n-1} \gamma_{i j} g^{i} x^{j}$. Since $h \cdot\left(\kappa^{C}(r)\right)=\sum h_{1}\left(\kappa^{C}(r)\right) S\left(h_{2}\right)$ for $h \in H$, we have that the equality $g \cdot\left(\kappa^{C}(r)\right)=\zeta^{-1} \kappa^{C}(r)$ implies that all terms equal zero except when $j=1$; hence

$$
\kappa^{C}(r)=\gamma_{0} x+\gamma_{1} g x+\cdots+\gamma_{n-1} g^{n-1} x
$$

for $\gamma_{i} \in k$. Also, the equality $x \cdot\left(\kappa^{C}(r)\right)=0$ implies that all terms equal zero except for $i=n-1$, so

$$
\kappa^{C}(r)=\gamma g^{n-1} x
$$

for $\gamma \in k$.

On the other hand, let $\kappa^{L}(r)=u \otimes f+v \otimes f^{\prime} \in V \otimes H$ for $f=\sum_{i, j=0}^{n-1} \lambda_{i j} g^{i} x^{j}$ and $f^{\prime}=\sum_{i, j=0}^{n-1} \lambda_{i j}^{\prime} g^{i} x^{j}$. Notice that $h \cdot\left(\kappa^{L}(r)\right)=\sum h_{1} \cdot u \otimes h_{2} f S\left(h_{3}\right)+$ $\sum h_{1} \cdot v \otimes h_{2} f^{\prime} S\left(h_{3}\right)$ (see Section 1A). Since $g \cdot\left(\kappa^{L}(r)\right)=\zeta^{-1} \kappa^{L}(r)$, all terms equal zero except possibly those in the first sum for which $j=1$ and those in the second sum for which $j=0$. Therefore $\kappa^{L}(r)=u \otimes f+v \otimes f^{\prime}$ for

$$
f=\lambda_{0} x+\lambda_{1} g x+\cdots+\lambda_{n-1} g^{n-1} x \quad \text { and } \quad f^{\prime}=\lambda_{0}^{\prime}+\lambda_{1}^{\prime} g+\cdots+\lambda_{n-1}^{\prime} g^{n-1}
$$

with $\lambda_{i}, \lambda_{i}^{\prime} \in k$. Applying $x$, we obtain

$$
\begin{aligned}
& 0=x \cdot \kappa^{L}(r)=(g \cdot u) \otimes(g f S(x)+x f S(1))+(x \cdot u) \otimes f \\
&+(g \cdot v) \otimes\left(g f^{\prime} S(x)+x f^{\prime} S(1)\right)+(x \cdot v) \otimes f^{\prime} \\
&=u \otimes\left(-g f g^{n-1} x+x f+f^{\prime}\right)+\zeta^{-1} v \otimes\left(-g f^{\prime} g^{n-1} x+x f^{\prime}\right) .
\end{aligned}
$$


It follows that

$$
-g f g^{n-1} x+x f+f^{\prime}=0 \text { and }-g f^{\prime} g^{n-1} x+x f^{\prime}=0 .
$$

Since $f^{\prime}$ is in the group algebra $k G(T(n)) \cong k \mathbb{Z}_{n}$ and $g^{n}=1$, the second equation implies that $x f^{\prime}=f^{\prime} x$, and so $f^{\prime}=\lambda_{0}$ is constant. The first equation further implies that $f^{\prime}=0$ and that all terms of $f$ are equal to zero except for $i=n-1$. Thus,

$$
\kappa^{L}(r)=u \otimes \lambda g^{n-1} x
$$

for $\lambda \in k$.

In summary,

$$
\mathscr{D}_{B, \kappa}=\frac{k\langle u, v\rangle \# T(n)}{(u v-v u-\kappa(u v-v u))}
$$

is a PBW deformation of $k[u, v] \# T(n)$ if and only if the deformation map $\kappa$ equals $\kappa^{C}+\kappa^{L}$ as given in (4-14) and (4-15). So, we have a two-parameter family of PBW deformations of $k[u, v] \# T(n)$.

Example 4.16. Let $H$ be $H_{S w}=T(2)$, the 4-dimensional nonsemisimple Sweedler algebra, which is a Taft algebra with $n=2$. Let $B=k[u, v]$. Retaining the notation from Example 4.13, the Hopf algebra $H_{S w}$ is generated by $g, x$ and acts on $B$ by $g \cdot u=u, g \cdot v=-v, x \cdot u=0, x \cdot v=u$. Similar to Example 4.13, let $r:=u v-v u$ and note that $g \cdot r=-r$ and $x \cdot r=0$. So, $I=\langle r\rangle$ is $H$-stable.

Let $\kappa^{C}(r)=\gamma_{0}+\gamma_{1} g+\gamma_{2} x+\gamma_{3} g x$. We have that $g \cdot\left(\kappa^{C}(r)\right)=-\kappa^{C}(r)$ implies that $\gamma_{0}=\gamma_{1}=0$. Moreover, $x \cdot\left(\kappa^{C}(r)\right)=0$ does not yield new restrictions on $\kappa^{C}(r)$. Thus, for $\gamma, \gamma^{\prime} \in k$, we get that $\kappa^{C}(r)=\gamma x+\gamma^{\prime} g x$. In the same fashion as Example 4.13, we also get that $\kappa^{L}(r)=u \otimes\left(\lambda x+\lambda^{\prime} g x\right)$ for $\lambda, \lambda^{\prime} \in k$.

In summary,

$$
\mathscr{D}_{B, \kappa}=\frac{k\langle u, v\rangle \# H_{S w}}{(u v-v u-\kappa(u v-v u))}
$$

is a PBW deformation of $k[u, v] \# H_{S w}$ if and only if the deformation map $\kappa$ equals $\kappa^{C}+\kappa^{L}$, where

$$
\kappa^{C}(u v-v u)=\gamma x+\gamma^{\prime} g x \text { and } \kappa^{L}(u v-v u)=u \otimes\left(\lambda x+\lambda^{\prime} g x\right)
$$

for $\gamma, \gamma^{\prime}, \lambda, \lambda^{\prime} \in k$. Thus, we have a four-parameter family of PBW deformations of $k[u, v] \# H_{S w}$.

Remark 4.17. The invariant ring resulting from the action of $H_{S w}$ on $k[u, v]$ is isomorphic to the polynomial ring $k\left[u, v^{2}\right]$, that is to say, $k[u, v]^{H_{S w}}$ is regular. Recall that the Shephard-Todd-Chevalley theorem states that when given a finite group $\left(G\right.$-) action on a commutative polynomial ring $R$ that is linear and faithful, $R^{G}$ is regular if and only if $G$ is a reflection group. Our results would then suggest that $H_{S w}$ is a "reflection Hopf algebra". Ram and Shepler [2003] showed that there are 
no nontrivial PBW deformations of $k\left[v_{1}, \ldots, v_{n}\right] \# k G$ for many complex reflection groups $G$; such deformations are referred to as graded Hecke algebras. Now by broadening their setting to Hopf actions on (possibly noncommutative) regular algebras, we consider new objects in representation theory: Hopf analogues of graded Hecke algebras. Nontrivial examples of these objects exist as we showed in the example above. Further examples and a general explanation of this phenomenon are worthy of further investigation.

Now we consider the well-known Hopf action of $U_{q}\left(\mathfrak{s l}_{2}\right)$ on $k\langle u, v\rangle /(u v-q v u)$, where $q \in k^{\times}$with $q^{2} \neq 1$. A PBW deformation of $(k\langle u, v\rangle /(u v-q v u)) \# U_{q}\left(\mathfrak{s l}_{2}\right)$ was studied by Gan and Khare [2007]; we recover their result below. Such algebras are known as quantized symplectic oscillator algebras of rank 1.

Example 4.18. Fix $q \in k^{\times}$, with $q^{2} \neq 1$. Let $H$ be the Hopf algebra $U_{q}\left(\mathfrak{s l}_{2}\right)$, and $B=k\langle u, v\rangle /(u v-q v u)$. As in [Brown and Goodearl 2002, I.6.2], we take $U_{q}\left(\mathfrak{s l}_{2}\right)$ to be generated by $E, F, K, K^{-1}$ with relations:

$$
\begin{gathered}
E F-F E=\left(q-q^{-1}\right)^{-1}\left(K-K^{-1}\right), \quad K E K^{-1}=q^{2} E, \\
K F K^{-1}=q^{-2} F, \quad K K^{-1}=K^{-1} K=1 .
\end{gathered}
$$

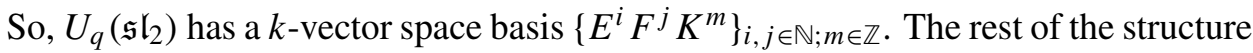
of $U_{q}\left(\mathfrak{s l}_{2}\right)$ and the left $U_{q}\left(\mathfrak{s l}_{2}\right)$-action on $B$ is given by:

$$
\begin{gathered}
\Delta(E)=E \otimes 1+K \otimes E, \quad \Delta(F)=F \otimes K^{-1}+1 \otimes F, \\
\Delta(K)=K \otimes K, \quad \Delta\left(K^{-1}\right)=K^{-1} \otimes K^{-1}, \\
\epsilon(E)=0, \quad \epsilon(F)=0, \quad \epsilon(K)=1, \quad \epsilon\left(K^{-1}\right)=1, \\
S(E)=-K^{-1} E, \quad S(F)=-F K, \quad S(K)=K^{-1}, \quad S\left(K^{-1}\right)=K, \\
E \cdot u=0, \quad F \cdot u=v, \quad K \cdot u=q u, \quad K^{-1} \cdot u=q^{-1} u, \\
E \cdot v=u, \quad F \cdot v=0, \quad K \cdot v=q^{-1} v, \quad K^{-1} \cdot v=q v .
\end{gathered}
$$

Let $r:=u v-q v u$ and note that $E \cdot r=F \cdot r=0$ and $K \cdot r=K^{-1} \cdot r=r$. Hence, the ideal of relations $I=\langle r\rangle$ of $B$ is $H$-stable.

Since $\operatorname{dim}_{k} V=2$, only condition (a) of Theorem 3.1 applies. Let us compute $\kappa^{C}(r)$. Since $K \cdot \kappa^{C}(r)=\kappa^{C}(K \cdot r)=\kappa^{C}(r)$, we have that $K \kappa^{C}(r) S(K)=\kappa^{C}(r)$ (see Section 1A). Hence, $K \kappa^{C}(r)=\kappa^{C}(r) K$. Moreover,

$$
0=\kappa^{C}(E \cdot r)=E \cdot \kappa^{C}(r)=E \kappa^{C}(r) S(1)+K \kappa^{C}(r) S(E),
$$

so $E \kappa^{C}(r)=\kappa^{C}(r) E$. Likewise, $F \cdot \kappa^{C}(r)=0$ implies that $F \kappa^{C}(r)=\kappa^{C}(r) F$. So, $\kappa^{C}(r)$ is in the center of $U_{q}\left(\mathfrak{s l}_{2}\right)$. For $q$ not a root of unity, the center of $U_{q}\left(\mathfrak{s l}_{2}\right)$ is 
generated by the quantum Casimir element [Kassel 1995, Theorem VI.4.8],

$$
C_{q}=E F+\frac{q^{-1} K+q K^{-1}}{\left(q-q^{-1}\right)^{2}}=F E+\frac{q K+q^{-1} K^{-1}}{\left(q-q^{-1}\right)^{2}},
$$

whereas for $q$ a root of unity, the elements $E^{e}, F^{e}, K^{e}$ also belong to the center of $U_{q}\left(\mathfrak{s l}_{2}\right)$, where $e=\operatorname{ord}\left(q^{2}\right)$.

To compute $\kappa^{L}(r)$, let $\kappa^{L}(r)=u \otimes \sum \gamma_{i j m} E^{i} F^{j} K^{m}+v \otimes \sum \gamma_{i j m}^{\prime} E^{i} F^{j} K^{m}$ for $\gamma_{i j m}, \gamma_{i j m}^{\prime} \in k$. Then,

$$
\begin{aligned}
\kappa^{L}(r) & =\kappa^{L}(K \cdot r) \\
& =\sum K \cdot u \otimes \gamma_{i j m} K\left(E^{i} F^{j} K^{m}\right) K^{-1}+\sum K \cdot v \otimes \gamma_{i j m}^{\prime} K\left(E^{i} F^{j} K^{m}\right) K^{-1} \\
& =\sum q u \otimes \gamma_{i j m} q^{2(i-j)} E^{i} F^{j} K^{m}+\sum q^{-1} v \otimes \gamma_{i j m}^{\prime} q^{2(i-j)} E^{i} F^{j} K^{m} \\
& =\sum q^{2(i-j)+1} u \otimes \gamma_{i j m} E^{i} F^{j} K^{m}+\sum q^{2(i-j)-1} v \otimes \gamma_{i j m}^{\prime} E^{i} F^{j} K^{m} .
\end{aligned}
$$

Thus, given $m \in \mathbb{Z} / n \mathbb{Z}$, define the subspace $V_{m} \subset U_{q}\left(\mathfrak{s l}_{2}\right)$ to be the $k$-span of all monomials $E^{i} F^{j} K^{\ell}$ such that $j-i \equiv m \bmod n$. Then $\kappa^{L}(u v-q v u) \in$ $u \otimes V_{2^{-1}}+v \otimes V_{-2^{-1}}$ if $q$ is a primitive root of unity of odd order, and $\kappa^{L}(u v-q v u)=$ 0 otherwise.

Therefore,

$$
\mathscr{D}_{B, \kappa}=\frac{k\langle u, v\rangle \# U_{q}\left(\mathfrak{s l}_{2}\right)}{(u v-q v u-\kappa(u v-q v u))}
$$

is a PBW deformation of $(k\langle u, v\rangle /(u v-q v u)) \# U_{q}\left(\mathfrak{s l}_{2}\right)$ if and only if $\kappa=\kappa^{C}+\kappa^{L}$, where $\kappa^{C}(u v-q v u)$ is in the center of $U_{q}\left(\mathfrak{s l}_{2}\right)$ and $\kappa^{L}(u v-q v u)$ is given as above.

More generally, there is a standard $U_{q}\left(\mathfrak{s l}_{n}\right)$-action on a $q$-polynomial ring $B$ in $n$ variables.

Question 4.19. Are there nontrivial PBW deformations of the resulting smash product algebra $B \# U_{q}\left(\mathfrak{s l}_{n}\right)$ ?

These would be quantized symplectic oscillator algebras of rank $n-1$, and merit further investigation.

\section{Acknowledgments}

Part of this work was completed during the spring 2013 program on Noncommutative Algebraic Geometry and Representation Theory at the Mathematical Sciences Research Institute. We thank the organizers for the facilities and generous hospitality. Walton and Witherspoon are supported by the National Science Foundation grants DMS-1102548 and DMS-1101399, respectively. 


\section{References}

[Berger and Taillefer 2007] R. Berger and R. Taillefer, "Poincaré-Birkhoff-Witt deformations of Calabi-Yau algebras", J. Noncommut. Geom. 1:2 (2007), 241-270. MR 2008i:16011 Zbl 1161.16022

[Braverman and Gaitsgory 1996] A. Braverman and D. Gaitsgory, "Poincaré-Birkhoff-Witt theorem for quadratic algebras of Koszul type", J. Algebra 181:2 (1996), 315-328. MR 96m:16012 Zbl 0860.17002

[Brown and Goodearl 2002] K. A. Brown and K. R. Goodearl, Lectures on algebraic quantum groups, Birkhäuser, Basel, 2002. MR 2003f:16067 Zbl 1027.17010

[Chan et al. 2012] K. Chan, E. Kirkman, C. Walton, and J. Zhang, Examples of actions of semisimple Hopf algebras on Artin-Schelter regular algebras, 2012. Private communication.

[Crawley-Boevey and Holland 1998] W. Crawley-Boevey and M. P. Holland, "Noncommutative deformations of Kleinian singularities", Duke Math. J. 92:3 (1998), 605-635. MR 99f:14003 Zbl 0974.16007

[Ding and Tsymbaliuk 2013] F. Ding and A. Tsymbaliuk, "Representations of infinitesimal Cherednik algebras", Represent. Theory 17 (2013), 557-583. MR 3123740

[Drinfeld 1986] V. G. Drinfeld, "Degenerate affine Hecke algebras and Yangians", Funktsional. Anal. i Prilozhen. 20:1 (1986), 69-70. In Russian; translated in Funct. Anal. Appl. 20:1 (1986), 58-60. MR 87m:22044 Zbl 0599.20049

[Etingof and Ginzburg 2002] P. Etingof and V. Ginzburg, "Symplectic reflection algebras, CalogeroMoser space, and deformed Harish-Chandra homomorphism", Invent. Math. 147:2 (2002), 243-348. MR 2003b:16021 Zbl 1061.16032

[Etingof et al. 2005] P. Etingof, W. L. Gan, and V. Ginzburg, "Continuous Hecke algebras", Transform. Groups 10:3-4 (2005), 423-447. MR 2006h:20006 Zbl 1115.20005

[Gan and Khare 2007] W. L. Gan and A. Khare, "Quantized symplectic oscillator algebras of rank one”, J. Algebra 310:2 (2007), 671-707. MR 2008c:17008 Zbl 1194.17003

[Gerstenhaber 1964] M. Gerstenhaber, "On the deformation of rings and algebras", Ann. of Math. (2) 79 (1964), 59-103. MR 30 \#2034 Zbl 0123.03101

[Guccione and Guccione 2002] J. A. Guccione and J. J. Guccione, "Hochschild (co)homology of Hopf crossed products”, K-Theory 25:2 (2002), 139-169. MR 2003h:16012 Zbl 1016.16005

[Kac and Paljutkin 1966] G. I. Kac and V. G. Paljutkin, "Finite ring groups", Trudy Moskov. Mat. Obšč. 15 (1966), 224-261. In Russian; translated in Trans. Moscow Math. Soc. 15 (1966), 251-294. MR 34 \#8211

[Kashina 2000] Y. Kashina, "Classification of semisimple Hopf algebras of dimension 16", J. Algebra 232:2 (2000), 617-663. MR 2001h:16041 Zbl 0969.16014

[Kassel 1995] C. Kassel, Quantum groups, Graduate Texts in Mathematics 155, Springer, New York, 1995. MR 96e:17041 Zbl 0808.17003

[Khare 2007] A. Khare, "Drinfeld-Hecke algebras over cocommutative algebras", preprint, 2007. arXiv 0705.2067

[Levandovskyy and Shepler 2014] V. Levandovskyy and A. V. Shepler, "Quantum Drinfeld Hecke algebras", Canad. J. Math. 66:4 (2014), 874-901. MR 3224268 Zbl 06331501

[Losev and Tsymbaliuk 2014] I. Losev and A. Tsymbaliuk, "Infinitesimal Cherednik algebras as W-algebras”, Transform. Groups 19:2 (2014), 495-526. MR 3200433 Zbl 06315919

[Lusztig 1989] G. Lusztig, "Affine Hecke algebras and their graded version”, J. Amer. Math. Soc. 2:3 (1989), 599-635. MR 90e:16049 Zbl 0715.22020 
[Masuoka 1995] A. Masuoka, "Semisimple Hopf algebras of dimension 6, 8", Israel J. Math. 92:1-3 (1995), 361-373. MR 96j:16045 Zbl 0839.16036

[McConnell and Robson 2001] J. C. McConnell and J. C. Robson, Noncommutative Noetherian rings, Graduate Studies in Mathematics 30, Amer. Math. Soc., Providence, RI, 2001. MR 2001i:16039 Zbl 0980.16019

[Montgomery 1993] S. Montgomery, Hopf algebras and their actions on rings, CBMS Regional Conference Series in Mathematics 82, Amer. Math. Soc., Providence, RI, 1993. MR 94i:16019 Zbl 0793.16029

[Naidu and Witherspoon 2014] D. Naidu and S. Witherspoon, "Hochshild cohomology and quantum Drinfeld Hecke algebras", preprint, 2014. arXiv 1111.5243

[Negron 2014] C. Negron, "Spectral sequences for the cohomology rings of a smash product", preprint, 2014. arXiv 1401.3551

[Norton 2013] E. Norton, "Symplectic reflection algebras in positive characteristic as Ore extensions", Preprint, 2013. arXiv 1302.5411

[Polishchuk and Positselski 2005] A. Polishchuk and L. Positselski, Quadratic algebras, University Lecture Series 37, Amer. Math. Soc., Providence, RI, 2005. MR 2006f:16043 Zbl 1145.16009

[Radford 2012] D. E. Radford, Hopf algebras, Series on Knots and Everything 49, World Scientific, Hackensack, NJ, 2012. MR 2894855 Zbl 1266.16036

[Ram and Shepler 2003] A. Ram and A. V. Shepler, "Classification of graded Hecke algebras for complex reflection groups", Comment. Math. Helv. 78:2 (2003), 308-334. MR 2004d:20007 Zbl 1063.20005

[Shepler and Witherspoon 2012a] A. V. Shepler and S. Witherspoon, "Drinfeld orbifold algebras", Pacific J. Math. 259:1 (2012), 161-193. MR 2988488 Zbl 1285.16026

[Shepler and Witherspoon 2012b] A. V. Shepler and S. Witherspoon, "A Poincaré-Birkoff-Witt theorem for quadratic algebras with group actions", preprint, 2012. To appear in Trans. Amer. Math. Soc. arXiv 1209.5660

[Shroff 2014] P. Shroff, “Quantum Drinfeld orbifold algebras”, preprint, 2014. arXiv 1304.6134

[Tikaradze 2010] A. Tikaradze, "Center of infinitesimal Cherednik algebras of $\mathfrak{g l}_{n}$ ", Represent. Theory 14 (2010), 1-8. MR 2011b:16111 Zbl 1250.17015

[Tikaradze and Khare 2010] A. Tikaradze and A. Khare, "Center and representations of infinitesimal Hecke algebras of $\mathfrak{s l}_{2}$ ”, Comm. Algebra 38:2 (2010), 405-439. MR 2011g:17024 Zbl 1282.17015

[Tsymbaliuk 2014] A. Tsymbaliuk, "Infinitesimal Hecke algebras of $\mathfrak{s o}_{n}$ ”, preprint, 2014. arXiv 1306.1514

[Weibel 1994] C. A. Weibel, An introduction to homological algebra, Cambridge Studies in Advanced Mathematics 38, Cambridge University Press, 1994. MR 95f:18001 Zbl 0797.18001

[Wu and Zhu 2013] Q. S. Wu and C. Zhu, "Poincaré-Birkhoff-Witt deformation of Koszul CalabiYau algebras”, Algebr. Represent. Theory 16:2 (2013), 405-420. MR 3035998 Zbl 1268.16030

Communicated by Georgia Benkart

Received 2013-12-18 Revised 2014-06-30 Accepted 2014-07-29

notlaw@math.mit.edu

Department of Mathematics, Massachusetts Institute of Technology, Cambridge, MA 02139, United States

sjw@math.tamu.edu

Department of Mathematics, Texas A\&M University, College Station, TX 77843, United States 


\section{Algebra \& Number Theory}

msp.org/ant

\section{EDITORS}

MANAGING EDITOR

Bjorn Poonen

Massachusetts Institute of Technology

Cambridge, USA

\author{
EDITORIAL BOARD CHAIR \\ David Eisenbud \\ University of California \\ Berkeley, USA
}

BOARD OF EDITORS

Georgia Benkart

Dave Benson

Richard E. Borcherds

John H. Coates

J-L. Colliot-Thélène

Brian D. Conrad

Hélène Esnault

Hubert Flenner

Edward Frenkel

Andrew Granville

Joseph Gubeladze

Roger Heath-Brown

Craig Huneke

János Kollár

Yuri Manin

Barry Mazur

Philippe Michel

Susan Montgomery
University of Wisconsin, Madison, USA

University of Aberdeen, Scotland

University of California, Berkeley, USA

University of Cambridge, UK

CNRS, Université Paris-Sud, France

University of Michigan, USA

Freie Universität Berlin, Germany

Ruhr-Universität, Germany

University of California, Berkeley, USA

Université de Montréal, Canada

San Francisco State University, USA

Oxford University, UK

University of Virginia, USA

Princeton University, USA

Northwestern University, USA

Harvard University, USA

École Polytechnique Fédérale de Lausanne

University of Southern California, USA
Shigefumi Mori

Raman Parimala

Jonathan Pila

Anand Pillay

Victor Reiner

Peter Sarnak

Joseph H. Silverman

Michael Singer

Vasudevan Srinivas

J. Toby Stafford

Bernd Sturmfels

Richard Taylor

Ravi Vakil

Michel van den Bergh

Marie-France Vignéras

Kei-Ichi Watanabe

Efim Zelmanov

Shou-Wu Zhang
RIMS, Kyoto University, Japan

Emory University, USA

University of Oxford, UK

University of Notre Dame, USA

University of Minnesota, USA

Princeton University, USA

Brown University, USA

North Carolina State University, USA

Tata Inst. of Fund. Research, India

University of Michigan, USA

University of California, Berkeley, USA

Harvard University, USA

Stanford University, USA

Hasselt University, Belgium

Université Paris VII, France

Nihon University, Japan

University of California, San Diego, USA

Princeton University, USA

\section{PRODUCTION}

production@msp.org

Silvio Levy, Scientific Editor

See inside back cover or msp.org/ant for submission instructions.

The subscription price for 2014 is US \$225/year for the electronic version, and $\$ 400 /$ year ( $\$ 55$, if shipping outside the US) for print and electronic. Subscriptions, requests for back issues and changes of subscribers address should be sent to MSP.

Algebra \& Number Theory (ISSN 1944-7833 electronic, 1937-0652 printed) at Mathematical Sciences Publishers, 798 Evans Hall \#3840, c/o University of California, Berkeley, CA 94720-3840 is published continuously online. Periodical rate postage paid at Berkeley, CA 94704, and additional mailing offices.

ANT peer review and production are managed by EditFLOW ${ }^{\circledR}$ from MSP.

\section{PUBLISHED BY}

mathematical sciences publishers

nonprofit scientific publishing

http://msp.org/

(C) 2014 Mathematical Sciences Publishers 


\section{Algebra \& Number Theory}

$\begin{array}{lll}\text { Volume } 8 & \text { No. } 7 \quad 2014\end{array}$

Double Dirichlet series and quantum unique ergodicity of weight one-half Eisenstein series

YiAnNis N. PETRIDis, Nicole RAUlF and Morten S. Ris AGER

Monodromy and local-global compatibility for $l=p$

ANA CARAIANI

Finite generation of the cohomology of some skew group algebras

VAN C. NGUYEN and SARAH WITHERSPOON

On the supersingular locus of the $G U(2,2)$ Shimura variety

BENJAMIN HOWARD and GEORgIOS PAPPAS

Poincaré-Birkhoff-Witt deformations of smash product algebras from Hopf actions on

1701

Koszul algebras

Chelsea Walton and SARAH Witherspoon

Highly biased prime number races

DANIEL FIORILLI

Bounded gaps between primes with a given primitive root

PAUL POLLACK 